\title{
Design and discovery of a highly potent ultralong- acting GLP-1 and glucagon co-agonist for attenuating renal fibrosis by modulating mitochondrial function
}

Xianxing Jiang ( $\square$ jiangxx5@mail.sysu.edu.cn )

Sun Yat-sen University https://orcid.org/0000-0002-7508-2368

Qian Zhao

Sun Yat-Sen University

Jiale Dong

Sun Yat-Sen University

Hui Chen

Sun Yat-Sen University

Huan Yu

Sun Yat-Sen University

Shuyin Ye

Shenzhen Turier Biotech. Co., Ltd.

Shuangjin Yu

The First Affiliated Hospital of Sun Yat-Sen University

Nazi Song

Sun Yat-Sen University

Hongjiao Xu

Sun Yat-sen University

Zhiteng Luo

Sun Yat-Sen University

Guodong Chen

The First Affiliated Hospital of Sun Yat-Sen University

Rui Wang

Lanzhou University https://orcid.org/0000-0002-4719-9921

Article

Keywords:

Posted Date: February 2nd, 2022 
DOI: https://doi.org/10.21203/rs.3.rs-1259932/v1

License: (c) (1) This work is licensed under a Creative Commons Attribution 4.0 International License. Read Full License 


\section{Abstract}

GLP-1 receptor (GLP-1R) and glucagon receptor (GCGR) are critical for the maintenance of glucose and energy homeostasis, but their roles in chronic kidney disease (CKD) are still unknown. Herein we found that the expression of GLP-1R and GCGR was lower in kidney from patients with CKD than in control individuals and was correlated with disease severity. RNA-seq analysis showed that adeno-associated viral vector serotype 9-GLP-1R or GCGR small hairpin RNA (AAV9-GLP-1R or GCGR shRNA) treatment in $\mathrm{db} / \mathrm{db}$ mice exhibited more advanced diabetic nephropathy than wild-type mice. We also found that GLP$1 \mathrm{R}$ mainly expressed in glomeruli and GCGR expressed in both glomeruli and tubuli renales. Based on the importance of GLP-1R and GCGR in CKD, we design and report a new monomeric peptide, 1907-B, with agonism at the GLP-1R and GCGR that have more prolonged half-time than long-acting semaglutide in cynomolgus rats or monkeys ( 2-3 fold) and exhibit better therapeutic contribution to CKD than best-inclass monoagonists, semaglutide or glucagon in $\mathrm{db} / \mathrm{db}$ mice and unilateral ureter obstruction (UUO) mice. Various loss-of-function models, including selective pharmacological blockade and genetic knockdown, confirmed that the effects of 1907-B on improving glucose control and ameliorating the diabetic nephropathy in $\mathrm{db} / \mathrm{db}$ mice were mediated through GLP-1 and glucagon signaling, whereas its action on inhibiting renal fibrosis in UUO mice was predominantly mediated through glucagon signaling. Mechanistically, 1907-B activated downstream cAMP-PKA pathway to promote mitochondrial biogenesis and bioenergetics, as well as to attenuate renal oxidative stress under chronic hyperglycemia via stimulating GLP-1R and GCGR simultaneously. Furthermore, 1907-B had crucial protection roles against inflammation via NFKB pathway and renal fibrosis via TGF- $\beta-S M A D$ pathway. Thus 1907-B, via direct kidney (GCGR) and part-glomerulus (GLP-1R) effects, exerts multifactorial improvement in renal injuries and is a more effective and promising therapeutic option for the treatment of CKD.

\section{Introduction}

Chronic kidney disease (CKD) characterized by persisting renal damage and /or loss of renal function is a severe disease with potentially high morbidity ${ }^{1,2}$. Chronic renal failure, as the end-stage renal disease, is manifested by glomerulosclerosis, tubulointerstitial fibrosis, and vascular sclerosis ${ }^{3,4}$. Unfortunately, the molecular mechanisms linking pathogenetic factors in kidney fibrosis are complicated and incompletely understood $^{5,6}$. It is most likely a combination of oxidative stress ${ }^{7-10}$, inflammation ${ }^{11-13}$, epithelial to mesenchymal transition, apoptosis ${ }^{14}$, and extracellular matrix deposition ${ }^{15}$. Thus, studies aimed at exploring attractive therapeutic targets and developing corresponding approaches for the treatment of CKD are urgently needed.

The mitochondrion is a central organelle that is responsible for the cellular redox, energy homeostasis, and therefore a major source of intracellular oxidative stress ${ }^{16}$. Recognition is growing that mitochondrial dysfunction and damages are recognized as a primary factor to various CKDs through impaired ATP generation, increased mitochondrial DNA (mtDNA) damage, and accumulated ROS production ${ }^{17-20}$. More recently, it has been demonstrated that ameliorating mitochondrial dysfunction prevents the progression 
of renal diseases ${ }^{21-23}$. Hence, mitochondria have been identified as an attractive platform for drug targeting in restraining CKD progression.

The protective role of GLP-1R signaling in diabetic nephropathy attributes to its hypoglycemic effect and anti-oxidative protective effect on various tissues ${ }^{24-27}$. On the other hand, recent evidence indicates that glucagon has lots of effects via a corresponding GCGR like improving mitochondrial turnover and function $^{28}$. At present, the application of GLP-1R/GCGR dual-target agonists in NASH ${ }^{29,30}$, obesity ${ }^{31-35}$ and diabetes ${ }^{36,37}$ has attracted extensive attention, such as Cotadutide (MEDI0382) ${ }^{38}, \mathrm{HM}-12525 \mathrm{~A}^{39}$, Oxyntomodulin (OPK-88003, TT401), BI456906 in phase II clinical trials. However, the therapeutic effects of these co-agonists on renal fibrosis remain unknown. In the context of this study, the emerging evidence indicated that levels of GLP-1R and GCGR in the kidney of human and experimental mice with CKD were lower than in healthy controls. Furthermore, GLP-1R and GCGR knockdown mice exhibited enhanced hyperglycemia-induced mitochondrial dysfunction, inflammation and renal fibrosis. Different from previous studies that only focused on the role of GLP-1R in CKD, we firstly found that GCGR also played a key role in the progression of CKD. The purpose of the current study was to follow up on this finding and develop a highly potent GLP-1 and glucagon co-agonist and further investigate its role in the CKD treatment.

Current GLP-1R/GCGR dual-target agonists regimens are potent and well-tolerated, enabling sustained suppression of many chronic and life-threatening disease. However, chronic disease control is linked to regimen adherence affected by pain and erythema at the site of injection. Such limitations were the impetus towards the development of ultralong-acting peptide drugs available ${ }^{40-45}$. To such ends, semaglutide once weekly is a successful attempt ${ }^{45-47}$. Despite such improvements, there remains no defined pathways to optimize prodrug hydrolysis and physicochemical features ${ }^{48}$. To this end, we firstly proposed the concept of "double-site recognition" via octadecanedioic (C18) and "GGSGSG" binding to albumin to optimize drug release. Through in vitro or in vivo screening, a high-potency, balanced coagonist 1907 for GLP-1R and GCGR was identified. Next, 1907-B with ultralong pharmacokinetic profiles was obtained by attaching an albumin-binding side chain derived from C18 and "GGSGSG" to the Lys10 of 1907 ( 2-3 fold to long-acting semaglutide in SD rats or cynomolgus monkeys).

Finally, we get a GLP-1R/GCGR dual-target agonist with ultralong-acting half-life, 1907-B, which prevents renal injury and fibrosis via anti-oxidative effect targeting GLP-1R and promoting mitochondrial turnover targeting GCGR. Furthermore, 1907-B notably relieves inflammation via NFKB pathway and improves renal fibrosis via TGF- $\beta$-SMAD pathway. In addition, CAMP-PKA pathway, the common downstream of GLP-1R and GCGR, plays a mechanistic role during 1907-B treatment by promoting mitochondrial biogenesis and bioenergetics. To our knowledge, no precedent has yet revealed the role of GLP-1R/GCGR dual targets, especially of GCGR target on attenuating renal fibrosis, and the current study provides an effective and promising alternative treatment strategy against renal fibrosis and CKD.

\section{Results}


GLP-1R and GCGR expression is correlated with progressive renal disease. We first detected the GLP-1R and GCGR levels of human kidney tissue in normal human or patients with diabetic nephropathy and other chronic kidney disease. The immunofluorescence staining results showed that GLP-1R was mainly expressed in the glomeruli and GCGR was expressed in whole kidney including renal tubules and glomeruli (Figure 1a). Moreover, we also found GLP-1R and GCGR levels were markedly lower in the kidneys of individuals with kidney disease than healthy individuals. Generally, GLP-1R and GCGR are closely correlated with metabolic disease. Consistently, less GLP-1R and GCGR levels were detected in kidney from patients with DN, even if the Remuzzi score (assessed the degree of chronic changes in glomeruli, tubules, interstitium, and blood vessels) of kidneys from patients with DN was lower than other patients with CKD (Figure 1b). The absence of GLP-1R and GCGR protein expression, as revealed by immunofluorescence analysis, was also found in diabetic nephropathy (DN) model in $\mathrm{db} / \mathrm{db}$ mice and renal fibrosis model in modelunilateral ureteral obstruction (UUO) mice (Figure 1c). Furthermore, qPCR analysis also confirmed that G/p1rand GcgrmRNA expression were also lower in the kidney of $\mathrm{db} / \mathrm{db}$ mice and UUO mice than in those of lean control group (Figure 1d). Collectively, the marked downregulation of GLP-1R and GCGR levels observed in renal disease indicates that they may have roles in the development of CKD.

To define the importance of GLP-1R and GCGR in progressive renal disease, we chose AAV9, which is used to transduce GLP-1R or GCGR shRNA to kidney, to down-regulate the expression of GLP-1R and GCGR in $\mathrm{db} / \mathrm{db}$ mice ${ }^{49}$. qPCR analysis indicated that a significant reduction of Glp1ror Gcgrexpression in the kidney was seen in the $\mathrm{db} / \mathrm{db}$ mice treated with AAV9-GLP-1R or GCGR shRNA (Supplementary Fig. 6d), together with much enhanced renal fibrosis (Figure 1e) and significant increase in mRNA expression of fibrotic factors (Figure 1f). Increased inflammation and impaired mitochondrial function were also observed in AAV9-GLP-1R or GCGR shRNA-infected mice but not control mice (Figure 1f). Thus, our findings demonstrated that GLP-1R and GCGR are key regulators in the progress of renal disease and can be used as the potential targets for the treatment of CKD.

GLP-1R and GCGR dual-agonist design. GLP-1 and glucagon bind to related members of the same receptor superfamily and exert important effects on glucose homeostasis, insulin secretion, and energy regulation. Therefore, combining the activity of two regulatory hormones with complementary biological effects, maybe offer a favorable strategy to treat diabetic nephropathy and related chronic kidney disease ${ }^{29}$.

To obtain just such a compound, we have designed seventeen novel GLP-1/glucagon chimeric peptides 1901-1917. These chimeric peptides have been created through fusion of the key amino acid sequences in maintaining biological activity of GLP-1 and glucagon (Supplementary Fig. $1 \mathrm{a}$ ) ${ }^{50}$. Comparing to the sequence information between GLP-1 and glucagon, positions 2, 3, 10, 12, 16, 20 are noteworthy in maintaining glucagon activity and positions 15, 18 are important in improving GLP-1R potency (Figure 2a). Considering that GLP-1 and glucagon commonly recognize their individual receptors through an ahelical conformation, we performed the amino acid substitutions in the middle of the peptides to stabilize the secondary structure and enhance binding affinities to related receptors. It has been identified that the 
salt bridges formed between 16-20 or 17-21 with specific helix-favoring amino acids in pairs could enhance helix stability through constrained conformation (Figure 2b). Moreover, glucagon achieves selectivity to GCGR due to its C-terminal acid. To reduce specificity for GCGR and activate GLP-1R more effectively, these chimeric peptides are designed to end with C-terminal amide. Besides, the C-terminal decapeptide from exendin-4 was assembled in the $\mathrm{C}$-terminus of chimeric peptides to further enhance GLP-1R efficacy.

Considering that N-terminal region of peptides is highly conserved, only modification occurs in the Ser2, which replaces with unnatural amino acid, D-Ser or 2-aminoisobutyric acid (Aib), to protect these peptides against the hydrolysis from DPPIV. Other kinds of alterations mainly occur in the middle and C-terminus of these peptides. In previous study, the fatty acid side chain has been reported to help these compounds to interact with the albumin and decrease their renal clearance. Based on the three-dimensional model of 1907/GLP-1R complex by homologous modeling (Figure 2c), we speculate that the introduction of fatty acid side chain at residue 10 or 14 of these chimeric peptides would probably not perturb receptor binding interaction ${ }^{50}$. Therefore, these 2 residues were used as modification sites for side chain to improve drug activity. In this study, high water solubility PEG spacer C18 was introduced to improve water solubility and prolong the half-lives of peptide analogues ${ }^{51}$.

Structural assessment and agonistic activity test of GLP-1R and GCGR dual-agonists. To study the structural conformation of these chimeric peptides, the circular dichroism (CD) spectra for each peptide was measured in an aqueous environment (PBS) and a membrane-mimicking environment (50\% TFE). As revealed in Supplementary Fig. 1b, these peptides showed random coil structures in aqueous buffer while displaying the typical a-helical spectra in 50\% TFE solution with two minimum peaks at 208 and $222 \mathrm{~nm}$. According to the Supplementary Table 1, GLP-1 analogues (liraglutide and semaglutide) and glucagon differ in primary as well as secondary structure and the differences in biological properties are likely a function of these secondary structural differences. The other hybrid peptides also differed appreciably in secondary structure in trifluoroethanol (TFE) concentrations of $50 \%(\mathrm{v} / \mathrm{v})$. The formation of salt bridges in these peptides and the Aib or Ser in position 16 are very important to the helical content of these peptides.

The agonistic activities among these peptide analogs were determined in HEK293 cells stably expressing human GLP-1R or GCGR using a cAMP response element (CRE)-driven luciferase reporter (Supplementary Table 2). Liraglutide, as a positive control of GLP-1R agonist, activated GLP-1R half maximally at an effective concentration ( $\mathrm{EC}_{50}$ ) of $0.04 \mathrm{nM}$ and activated GCGR with an $\mathrm{EC}_{50}$ exceeding $1 \mu \mathrm{M}$. Native glucagon activated GCGR at an $\mathrm{EC}_{50}$ of $111.45 \mathrm{nM}$ and activated GLP-1R with an $\mathrm{EC}_{50}$ exceeding $1 \mu \mathrm{M}$. Other chimeric peptides also showed excellent GLP-1R agonism with the $\mathrm{EC}_{50}$ of $0.002-0.091 \mathrm{nM}$. Meanwhile, the incorporation of glucagon residues gives part of chimeric peptides the ability to activate GCGR with the $\mathrm{EC}_{50}$ of $69.66-337.70 \mathrm{nM}$. The replacement of unnatural amino acid, D-Ser or Aib, at position 2 provided peptides with enhanced stability against DPPIV, but did not influence in vitro profile remarkably. Moreover, replacing Glu with Asp in position 15 and the replacement of Glu in position 16 did 
enhance GCGR efficacy ( 5-fold) without altering GLP-1R activity. Arg substitution at position 27 of the peptide 1909 resulted in the highest a-helical content and lowest sensitivity to GLP-1R with an $\mathrm{EC}_{50}$ of $0.838 \mathrm{nM}$ among these peptides, indicating that the original residue plays a vital role in activating GLP$1 \mathrm{R}$.

GLP-1R and GCGR dual-agonist screening in vitro and in vivo. With these highly potent GLP-1 and glucagon co-agonist analogs in hand, we next explored the efficacy of combined GLP-1 and glucagon agonism in a single peptide. Hyperglycemia is a main performance of diabetes and affects many intracellular processes. Diabetic nephropathy characterized by glomerular mesangial expansion and tubular epithelial-mesenchymal transition (EMT) induced by hyperglycemia, could result in increased cell extracellular matrix synthesis and further renal fibrosis formation ${ }^{52,53}$. To evaluate the therapeutic effects of these hybrid peptides on progressive renal disease, high glucose-induced GMC (glomerular mesangial cells) model and mTEC (tubular epithelial cells) model were used in vitro studies (Supplementary Fig. 1c and 1d). Fibronectin (FN) is an extracellular matrix component and is present along glomerular basement membranes. During glomerular injury, the deposition of FN would increase. Thus, FN is used as a marker of diabetic glomerular injury. Western blotting assay confirmed that these analogues could significantly decrease the expression level of FN in high glucose induced GMC cells. Moreover, alpha smooth muscle actin (a-SMA) level from the kidney areas increases with ECM deposition, and this is a hint that indicates alteration in myofibroblasts. Western blotting assay showed that part of analogues, especially peptides with balanced GLP-1R and GCGR agonism could obviously reduce the a-SMA level in the high glucose induced mTEC cells. These results were consistent with the previous findings, suggesting that the impact of these peptides on glomeruli outcomes was mediated through GLP-1R and GCGR signaling in glomeruli, while other impact on tubules directly mediated through GCGR signaling in tubules.

Next, we performed glucose tolerance test in vivo at different time points after peptides treatment to assess peptides response to glucose and their long-acting potency. Liraglutide and semaglutide are used as the positive control groups because of their long half-life. As shown in Supplementary Fig. 2, blood glucose levels of these peptides treated mice were significantly lower than those of PBS treated control mice after administration $8 \mathrm{~h}$ and $12 \mathrm{~h}$ (Supplementary Fig. $2 \mathrm{a}$ and $2 \mathrm{~b}$ ). Among them, the glucose tolerance improvement effects of 1907 and 1917 were more obvious than other peptides analogues. Even after 1907 and 1917 treatment for $24 \mathrm{~h}$, the blood glucose level still could be maintained in a proper range as same as semaglutide treatment, and their effects on glucose control were better than that of liraglutide (Supplementary Fig. 2c). It was stated clearly that 1907 and 1917 had relatively great half-time and could be used for the further evaluation of therapeutic effect on progressive renal disease in vivo.

1907 and 1917 generate synergistic metabolic benefits and ameliorate renal injury in $\mathrm{db} / \mathrm{db}$ mice. The $\mathrm{db} / \mathrm{db}$ mice were chosen in the present study because they could spontaneously develop detectable $\mathrm{DN}^{54}$. Before evaluating the therapeutic effects of 1907 and 1917 on DN, the optimal doses of 1907 and 1917 were explored between 80, 100 and $120 \mu \mathrm{g} / \mathrm{kg}$, and $120 \mu \mathrm{g} / \mathrm{kg}$ performed best (Supplementary Fig. 3b-3d). Semaglutide was included in the assay with $120 \mu \mathrm{g} / \mathrm{kg}$ as an optimal dose (Supplementary Fig. 3a). 12-week-old male db/db mice suffered from 1907, 1917 or semaglutide treatment for 8 weeks 
(Figure $2 \mathrm{~d}$ ), while $\mathrm{db} / \mathrm{m}$ mice served as the nondiabetic controls. At 12 weeks of age, $\mathrm{db} / \mathrm{db}$ mice became more obese than $\mathrm{db} / \mathrm{m}$ mice. After 8 weeks of treatment, compared to those of the control $\mathrm{db} / \mathrm{db}$ mice, 1907, 1917 and semaglutide-treated db/db mice showed obviously lower serum lipid (TC and TG) levels (Supplementary Fig. 4e). H\&E staining exhibited that hepatocyte injury due to lipotoxicity was observed in $\mathrm{db} / \mathrm{db}$ mice; whereas, 1907, 1917 and semaglutide treatment markedly reduced the ballooning and hepatic steatosis in the mice liver (Supplementary Fig. 4a). Moreover, 1907 significantly decreased the body weight, liver weight and epididymis fat weight via suppressing food intake, water intake and promoting lipolysis in liver compared to vehicle control (Figure 2e and Supplementary Fig. 4). In contrast, 1917 and semaglutide showed no obvious decline in obesity levels. In addition, levels of serum ALT and AST were remarkably reduced in the 1907 treated $\mathrm{db} / \mathrm{db}$ mice, as compared to the other group, which indicated the excellent liver protection function (Supplementary Fig. 4e). Collectively, these data suggested that 1907 treatment promoted lipid metabolism benefits and protected mice against lipid dysfunction.

The $\mathrm{db} / \mathrm{db}$ mice exhibited higher fasting blood glucose levels when compared to $\mathrm{db} / \mathrm{m}$ mice during the 8week experiment (as shown in Figure 2e), whereas the 1907, 1917 and semaglutide-treated groups showed lower fasting glucose levels than vehicle control. In addition, 1907, 1917 and semaglutide treatment significantly reduced hyperglycemia-induced glucose intolerance and insulin resistance, as assessed by OGTT and ITT, and among them, 1907 improved the insulin resistance best (Figure $2 \mathrm{f}$ and $2 \mathrm{~g}$ ). Furthermore, compared with $\mathrm{db} / \mathrm{m}$ mice, $\mathrm{db} / \mathrm{db}$ mice obviously affected the islet topography and the number of insulins ${ }^{+}$cells. However, 1907, 1917 and semaglutide treatment could significantly improve islet and promote the secretion of insulin (Supplementary Fig. 5c). These data suggested that 1907, 1917 and semaglutide promoted glucose metabolism benefits and protected mice against the damage from hyperglycemia.

The $\mathrm{db} / \mathrm{db}$ mice showed significantly increased serum creatinine $(\mathrm{SCr})$ and blood urea nitrogen (BUN) compared with the control mice (Figure 2h). Additions of 1907, 1917 and semaglutide significantly reduce $\mathrm{BUN}$ and $\mathrm{SCr}$ in the $\mathrm{db} / \mathrm{db}$ mice. Microalbuminuria is considered the earliest clinical indicator in the early stage of $\mathrm{DN}^{55}$. As shown in Figure $2 \mathrm{~h}$, albuminuria was significantly decreased in the 1907 , 1917 and semaglutide-treated diabetic $\mathrm{db} / \mathrm{db}$ mice compared with $\mathrm{db} / \mathrm{db}$ control mice. Meanwhile, urine glucose was also obviously decreased under the 1907, 1917 and semaglutide treatment. Furthermore, the renal histopathology analysis was performed and the results indicated that the $\mathrm{db} / \mathrm{db}$ mice displayed kidney damage and severe inflammatory infiltration (Supplementary Fig. 5a), while administration of 1907, 1917 and semaglutide resulted in a marked decrease in renal injury by changes in kidney sections stained with H\&E (Figure $2 \mathrm{i}$ ). Compared with $\mathrm{db} / \mathrm{m}$ mice, the $\mathrm{db} / \mathrm{db}$ mice progressed with glomerular hypertrophy and mesangial matrix expansion as PAS results. 1907, 1917 and semaglutide treatment maintained the renal glomerular structure and reduced the glomerular mesangial matrix accumulation compared to the $\mathrm{db} / \mathrm{db}$ mice. Moreover, accumulation of collagen fibers was also reduced in the 1907 , 1917 and semaglutide treatment groups compared to the $\mathrm{db} / \mathrm{db}$ mice (Figure 2i and Supplementary Fig. 5b). TGF- $\beta 1$, as one of crucial mediators for excessive ECM deposition in DN, could give rise to 
glomerulosclerosis ${ }^{56}$. In this study, immunohistochemistry was performed to assess the level of TGF- $\beta 1$ in glomeruli of $\mathrm{db} / \mathrm{db}$ mice (Supplementary Fig. $5 b$ ). A significant increase for the positive staining of TGF- $\beta 1$ in the glomeruli was observed in the $\mathrm{db} / \mathrm{db}$ mice compared to the normal control mice. Whereas, glomerular staining of TGF- $\beta 1$ was efficiently abrogated in db/db mice treated with 1907, 1917 and semaglutide. Combined, these observations indicated that 1907, 1917 and semaglutide could decrease the collagen deposition, thereby ameliorating renal fibrosis.

A rationally designed 1907 analogue, 1907-B, exhibits an extended half-life in vivo. Based on above data, the analogue 1907 was finally picked up for further modification to obtain a highly potent ultralongacting GLP-1R and GCGR co-agonist with sufficient bioavailability. In preliminary study, we found that GCGR played a vital role in the progression of CKD and appropriately increasing the affinity of peptides to GCGR may be more conducive to the treatment of CKD. Therefore, the C-terminal amide bond of 1907 was replaced with a C-terminal acid (Figure 3a). Moreover, our study revealed the fatty acid side chain, C18 helped the 1907 to interact with the albumin and prolonged its half-time to $24 \mathrm{~h}$ or even longer. Considering that "GGSGSG" also could bind to the albumin, we hypothesized that strong and reversible binding to albumin by the combination of C18 and "GGSGSG" in the side chain of Lys at position 10 of 1907(as 1907-B), would mediate increased stability toward proteolysis and lead to 1907-B with desired properties (Figure 3b). As our strategy about enhancing albumin affinity to obtain protraction, 1907 or 1907-B binding to the human serum albumin (HSA) was evaluated in biosensor experiments with surface plasmon resonance (SPR). The sensorgrams showed that 1907-B exhibited $>10$-fold higher affinity binding to HSA $(K D=2.54 \mu \mathrm{M})$ than $1907(K D=26.5 \mu \mathrm{M})$ (Figure 3c). Consistent with above result, isothermal titration calorimetry (ITC) assay also identified that 1907-B could increase the binding affinity with 4-fold compared with 1907 (Figure 3d). Furthermore, to confirm the role of "GGSGSG" in increasing the binding affinity with HSA, tetraethylene glycol or "GGSGSG" was tested by ITC. The results exhibited that "GGSGSG" had high binding affinities with $K D=866 \mu \mathrm{M}$, whereas tetraethylene glycol showed no HSA binding. Next, the changes of structural conformation and agonistic activities on GLP-1R or GCGR between 1907 and 1907-B were examined. The results indicated that the additional modification on 1907 could raise a-helical content, decrease the GLP-1R activity ( 44-fold) and increase GCGR activity ( 10fold) to promote more balanced GLP-1R/GCGR activity ratio (Figure $3 e$ ).

On the basis of the enhanced binding affinity between 1907-B and HSA, we need to identify if the halftime of 1907-B could be prolonged. After 1907-B treatment for $60 \mathrm{~h}$, the OGTT results showed that 1907-B still obviously improved the glucose tolerance compared with saline group. In comparison, the 1907 and semaglutide robustly lowered glucose excursion after treatment for $48 \mathrm{~h}$ (Figure 3f). Next, we evaluated the PK properties of 1907-B and semaglutide by intravenous injection or subcutaneous injection in SD rats. According to the Figure $3 g$, following i.v. treatment, the half-lives $\left(t_{1 / 2}\right)$ of 1907-B and semagoutide were $\sim 16.8 \mathrm{~h}$ and $\sim 8 \mathrm{~h}$, accordingly. The observed AUCs from 0 to infinity (AUCinf) of 1907-B and semaglutide were $\sim 8588$ and $\sim 6194 \mathrm{ng} \cdot \mathrm{h} / \mathrm{mL}$, respectively. Following s.c. treatment, $\mathrm{t}_{1 / 2}$ of 1907-B and semaglutide were $\sim 20.5 \mathrm{~h}$ and $\sim 7.95 \mathrm{~h}$, accordingly. Meanwhile, we also assessed the PK properties of 1907-B in cynomolgus monkeys (Figure 3h). Following s.c. treatment, the $t_{1 / 2}$ of 1907-B was $84 \mathrm{~h}$ and the 
$t_{1 / 2}$ of reported semaglutide was $53 \mathrm{~h}$. These data indicated that the $t_{1 / 2}$ of 1907-B was prolonged to $\sim 2-$ 3 -fold than that of semaglutide, which has a dosing frequency of once weekly in humans. These results pointed toward 1907-B, a ultralong-acting peptide, having a potential possibility that can be administered once every two weeks or even less frequency.

Toxicological evaluation of 1907-B in SD rats. The toxicity level of 1907-B was also evaluated in SD rats with low dose $(0.25 \mathrm{mg} / \mathrm{kg})$, medium dose $(0.75 \mathrm{mg} / \mathrm{kg})$ and high dose $(1.5 \mathrm{mg} / \mathrm{kg})$. The results indicated that 1907-B reduced the body weight in a dose-dependent manner because of the GCG-signaling component of 1907-B (Supplementary Table 3-4). Besides, there was no significant abnormalities in general behavior, appetite, physical appearance and various hematology (Supplementary Table 5-8). Meanwhile, pathological analysis showed that 1907-B did not cause obvious pathological changes on the organs (liver, spleen, lung, kidney, stomach, duodenum, uterus, ovary, testis and epididymis) of rats under high dose (Supplementary Fig. 8). Taken together, 1907-B has great safety and can be used for further research.

1907-B improves renal injury depend on GLP-1R and GCGR signaling in db/db mice. To directly examine the in vivo efficacy of ultralong-acting 1907-B, we performed a series of dose and administration frequency of $1907-B$ in db/db mice with diabetic nephropathy $(60,120,240 \mu \mathrm{g} / \mathrm{kg} / \mathrm{day}$ or $120 \mu \mathrm{g} / \mathrm{kg} / 2$ days). The blood glucose changes showed 1907-B dramatically decreased the blood glucose level in a dose dependent manner and even revealed better efficacy than semaglutide in $\mathrm{db} / \mathrm{db}$ mice (120 and 240 $\mu \mathrm{g} / \mathrm{kg} /$ day) (Figure 4a). Meanwhile, we also measured the $\mathrm{HbA1c}$ levels of each group after 1907-B treatment for 12 weeks. Consist with above results, 1907-B also could significantly decrease the HbA1c with dose dependency and 120 and $240 \mu \mathrm{g} / \mathrm{kg} /$ day groups showed better effect in reducing the HbA1c than semaglutide. Based on the principle that the lowest dose and the lowest frequency were given priority under ensuring the efficacy, we chose $1907-B$ with $120 \mu \mathrm{g} / \mathrm{kg} / 2 \mathrm{~d}$, which is equivalent to the effect of semaglutide for the further study.

Metabolic and kidney outcomes of 1907-B administration were evaluated in vivo using $\mathrm{db} / \mathrm{db}$ mice accompanied by diabetic nephropathy. Following 8 weeks of equivalent dosing $(120 \mu \mathrm{g} / \mathrm{kg})$ with 1907-B (dual GLP-1R/GCGR agonist) once every 2 days, semaglutide (GLP-1R agonist) daily or glucagon (GCGR agonist) daily, there was no difference for weight-lowering effects between the treatment groups and the vehicle group (Supplementary Fig. 6a). Fasting glucose level was decreased in db/db mice with 1907-B and semaglutide treatment compared with vehicle, in contrast, glucagon alone induced higher fasting glucose level (Supplementary Fig. 6b). Meanwhile, the HbA1c level was obviously decrease in 1907-B treatment, but not in semaglutide and glucagon treated $\mathrm{db} / \mathrm{db}$ mice. Interestingly, although treatment with the glucagon resulted in much higher blood glucose, it still exhibited the similar effect on reducing BUN and $\mathrm{SCr}$ as 1907-B and semaglutide (Figure 4b). This effect was also identified in pathological results. As shown in Figure 4c, H\&E, Masson and Sirius Red staining showed that $\mathrm{db} / \mathrm{db}$ mice exhibited more obvious glomerulosclerosis and tubulointerstitial fibrosis than the control group. However, 1907-B, semaglutide and glucagon treatment could significantly decrease glomerulosclerosis and tubulointerstitial fibrosis in the $\mathrm{db} / \mathrm{db}$ mice. To systematically explore the effects of 1907-B, semaglutide 
and glucagon on DN, the RNA-seq analysis were performed. Gene Set Enrichment Analysis (GSEA) pathway enrichment results indicated that enriched pathways involved in fibrosis and inflammation were downregulated and enriched pathway involved in mitochondrial function was upregulated by 1907-B, semaglutide and glucagon treatment compared with the control $\mathrm{db} / \mathrm{db}$ mice (Figure $4 \mathrm{e}$ and Supplementary Fig. 6c). A heatmap based on the GSEA results indicated that genes involved in fibrosis and inflammation were downregulated and genes involved in mitochondrial function were upregulated by 1907-B, semaglutide and glucagon treatment compared with the control db/db mice (Figure 4d). These data demonstrate both GLP-1R and GCGR played the vital roles in DN and 1907-B improved kidney function and inhibited renal fibrosis via targeting GLP-1R and GCGR.

To evaluate whether GLP-1R and GCGR signaling were required for 1907-B to attenuate renal damage and fibrosis, we chose adeno-associated viral (AAV) system, AAV9 vectors to deliver GLP-1R or GCGR shRNA to $\mathrm{db} / \mathrm{db}$ mice with diabetic nephropathy $\left(\mathrm{GLP}-1 \mathrm{R}^{-/-}\right.$or $\mathrm{GCGR}^{-/-}$) (Supplementary Fig. 6e). Four weeks after the AAV9 injection, db/db mice with the GLP-1R or GCGR knockdown showed high fasting blood glucose as control db/db mice (Figure 4f). Blood glucose-lowering effect of 1907-B in db/db mice lacking GLP-1R was not observed, while 1907-B treatment in $\mathrm{db} / \mathrm{db}$ mice lacking GCGR showed the most obvious blood glucose-lowering effect. There was no difference for $\mathrm{HbA} 1 \mathrm{C}$ and $\mathrm{SCr}$ levels between GLP-1R or GCGR knockdown and control db/db mice after 1907-B treatment (Figure $4 \mathrm{f}$ and $4 \mathrm{~g}$ ). Interestingly, GCGR knockdown could markedly lower the potency of 1907-B in reducing BUN, while 1907-B still significantly decreased the BUN under GLP-1R knockdown. Consist with above results, there was no effect on glomerulosclerosis and tubulointerstitial fibrosis with 1907-B treatment in GCGR knockdown db/db mice via H\&E, Masson and Sirius Red staining (Figure 4h). In contrast, 1907-B treatment could significantly improve glomerulosclerosis and tubulointerstitial fibrosis in GLP-1R knockdown $\mathrm{db} / \mathrm{db}$ mice. These findings further proved 1907-B improved DN via targeting GLP-1R and GCGR, meanwhile GCGR plays a more important role than GLP-1R during treatment.

1907-B attenuates renal fibrosis mainly depend on GCGR signaling in UUO mice. The UUO method, most commonly of the left one, has been widely applied to establish animal models with obstructive nephropathy via the ureter ligation (Supplementary Fig. 6f) ${ }^{57}$. H\&E, Masson and Sirius Red staining were performed to assess kidney injury and fibrosis. Histologically, compared with sham group, UUO group is characterized by tubular dilation and atrophy, renal parenchyma loss, inflammatory cells infiltration, and extracellular matrix (ECM) accumulation. In order to obtain the optimal efficiency, we performed a series of dose and administration frequency of 1907-B in UUO mice $(60,120,240 \mu \mathrm{g} / \mathrm{kg} /$ day or $120 \mu \mathrm{g} / \mathrm{kg} / 2$ days). Similarly, the pathological results indicated that 1907-B dramatically reduced renal inflammation and fibrosis in UUO mice in a dose dependent manner and even exhibited better efficacy than semaglutide (Figure 5a). Based on these results, we chose the $120 \mu \mathrm{g} / \mathrm{kg} / 2$ days for the further study.

The effect of 1907-B treatment on kidney fibrosis improvement were assessed in vivo using UUO mice accompanied by renal fibrosis. Following 14 days of equivalent dosing $(120 \mu \mathrm{g} / \mathrm{kg})$ with $1907-\mathrm{B}$ once every 2 days, semaglutide daily or glucagon daily, H\&E, Masson and Sirius Red staining revealed that glucagon treatment exhibited markedly fewer tubular injuries and ECM accumulation than 1907-B and 
semaglutide treatment (Figure 5b). To further systematically investigate how 1907-B, semaglutide and glucagon suppress UUO-induced renal fibrosis, we performed RNA-seq analysis in the kidney from sham and UUO-treated mice. GSEA systematically clarified that cellular signaling pathways involved in fibrosis, inflammation and mitochondrial function were enriched (Supplementary Fig. 6h). Similarly, a heatmap based on GSEA revealed that the expression of renal genes involved in fibrosis and inflammation was significantly downregulated and the expression of renal genes involved in mitochondrial function was upregulated by 1907-B and glucagon in UUO mice, compared with semaglutide treatment (Figure 5c). Subsequently, the data in combination with the transcriptomes of DN and UUO mice were analyzed, which demonstrated that nine signaling pathways were consistently influenced (Figure 5d), and the inflammation and mitochondrial function pathways were overlapped (Figure 5e).

To further confirm the role of GLP-1R and GCGR during the 1907-B treatment for renal fibrosis, we generated AAV9-GLP-1R or GCGR shRNA treated UUO mice (Supplementary Fig. 6g). H\&E, Masson and Sirius Red staining revealed that there was no difference for the renal fibrosis between the UUO mice and the GLP-1R or GCGR knockdown UUO mice. Improved kidney damage of 1907-B in UUO mice lacking GCGR were not observed, while 1907-B treatment in UUO mice lacking GLP-1R showed obvious renal protection effects, including fewer tubular injuries and ECM deposition (Figure 5f). These findings further proved 1907-B improved renal fibrosis in UUO mice mainly via targeting GCGR.

\section{7-B ameliorates renal inflammation and fibrosis via IKBa-NF-KB and TGF- $\beta 1-S M A D$ signaling}

pathways. Next, we used the DN model as the research object to explore the mechanism of 1907-B treatment on progressive renal disease. According to RNA-seq analysis results, the cellular signaling pathways involved in fibrosis (such as VEGF signaling pathway and ECM receptor interaction) were significantly downregulated by 1907-B treatment (Figure 6a). Western blot detection similarly revealed 1907-B treatment reduced a-SMA and COL1A1 expression in db/db mice (Figure 6b). Furthermore, qPCR results also indicated that 1907-B treatment could suppress the genes expression related to fibrosis, such as Serpine1, Desmin, Mmp3, Mmp8, and Mmp9, suggesting that 1907-B treatment may not only promote ECM degradation, but also decrease ECM production (Figure $6 \mathrm{c}$ ). It is well known to all that TGF- $\beta / S M A D$ signaling pathway is pivotal in the formation of renal fibrosis ${ }^{58}$, we then investigated whether 1907-B could modulate the TGF- $\beta /$ SMAD signaling pathway to ameliorate renal fibrosis. As showed in Figure $6 \mathrm{~b}$, the expression of pro-fibrogenic gene TGF- $\beta 1$ as well as phosphorylated SMAD2/3 in db/db mice were downregulated by 1907-B treatment. Considering that fibroblast activation plays a major role for kidney interstitial fibrosis and activated fibroblasts or myofibroblasts are sources of ECM. We evaluated the impact of 1907-B on fibroblast activation using high glucose-treated GMC and mTEC cells. Similarly, the pro-fibrogenic genes such as TGF- $\beta 1$ in high glucose-induced GMC and mTEC cells were also obviously downregulated by 1907-B treatment (Figure 6d).

Inflammation is a hallmark of progressive renal disease characterized by inflammatory cytokine production and inflammatory cell infiltration. Next, we determined the effects of 1907-B on DN-associated inflammation. RNA-seq analysis results showed the cellular signaling pathways related to inflammation (such as cytokine-cytokine receptor interaction, chemokine signaling pathway and toll like receptor 
signaling et.al) were significantly downregulated by 1907-B treatment (Figure 6e). qPCR assay also showed that 1907-B treatment obviously attenuated the increase in the expression of inflammation genes (Nos2, F4/80, I/1b, I/6, Cc/2 and Cc/4) in db/db mice (Figure 6f). The anti-inflammatory effect of 1907-B treatment was also revealed by reductions in inflammatory cell infiltration from the kidney, as verified by immunohistochemistry staining for the macrophage marker CD68 (Figure 6g). Similarly, western blot detection also identified that 1907-B treatment could significantly decrease the expression of the inflammation genes (CD68, TNF-a and IL-1 $\beta$ ) (Figure 6h). NF-KB signaling is activated in $\mathrm{db} / \mathrm{db}$ mice with DN. Western blot detection exhibited that the protein levels of $\mathrm{p}-\mathrm{IKBa}$ and $\mathrm{p}-\mathrm{P} 65$ were obviously decreased by 1907-B treatment (Figure 6h). Consist with the results in $\mathrm{db} / \mathrm{db}$ mice, western blot detection in high glucose-induced GMC and mTEC cells also showed that the protein levels of $\mathrm{p}$-IKBa and p-P65 were remarkably reduced by 1907-B treatment, whereas those of IKBa were increased by 1907-B, suggesting that 1907-B suppresses the activation of NF-KB signaling and related inflammation (Figure 6i).

1907-B improves mitochondrial function and inhibits oxidative stress. To explore the potential effect by which 1907-B protects against progressive renal disease, we further analyzed the transcriptomes of $\mathrm{db} / \mathrm{db}$ mice treated with 1907-B. RNA-Seq analysis exhibited that 1907-B treatment influenced diverse signaling pathways involved in metabolism, such as butanoate metabolism, citrate cycle TCA cycle, peroxisome and oxidative phosphorylation (Figure 7a). Oxidative stress was detected by determination of levels of SOD, CAT, HO-1 and the activity of GSH, CAT, T-SOD in renal tissues. These data showed 1907-B treatment could remarkably ameliorate levels and activity of these antioxidases in $\mathrm{db} / \mathrm{db}$ mice (Figure $7 \mathrm{~b}$ and 7c). In addition, we also examined the levels of 4-HNE and NOX4 in db/db mice after 1907-B treatment using immunofluorescence staining and the results showed 1907-B treatment significantly reduced the contents of $4-\mathrm{HNE}$ and NOX4 in $\mathrm{db} / \mathrm{db}$ mice (Figure 7d). The similar results also were observed by western blot detection in $\mathrm{db} / \mathrm{db}$ mice or in high glucose-induced GMC and MTEC cells after 1907-B treatment (Figure 7e and 7g). Furthermore, we evaluated the effect of 1907-B on the improvement of mitochondrial function. QPCR analysis showed that 1907-B could increase the expression of genes related to mitochondrial function, such as Ppargc1a, Ppargc1b, Uqcrc1 and Gpx1. Similarly, western blot detection also identified that 1907-B could increase the expression of proteins, such as Ppargc1a and Ppargc $1 b$ in db/db mice or in high glucose-induced GMC and mTEC cells after 1907-B treatment (Figure $7 \mathrm{f}$ and $7 \mathrm{~h}$ ). These findings indicate that 1907-B could decrease the oxidative stress and ameliorate mitochondrial function to further improve renal disease.

1907-B enhances mitochondrial function via PKA signaling. Mitochondrial dysfunction has been implicated in diabetic nephropathy pathogenesis. Whether 1907-B could alter mitochondrial dynamics and function was performed in high glucose induced-GMC and mTEC cells. Decreased number of greenlabelled mitochondria was observed in high glucose-treated cells compared with low glucose-treated group (Figure 8a and 8b). In contrast, 1907-B could significantly recover the mitochondrial dynamics induced by high glucose. Mitochondrial fission is often used for the determinant of mitochondrial function ${ }^{59}$ and the mitochondrial length was examined to quantify mitochondrial fission. We observed the morphological changes in mitochondria under high glucose treatment. and the mitochondrial length 
was significantly reduced compared with control cells. However, 1907-B treated cells showed predominantly elongated forms of mitochondria despite treatment with high glucose. The inhibition of PKA could abolished the improvement in mitochondrial maintenance by 1907-B.

Next, we used oxygen-consumption rate (OCR) assay to evaluate mitochondrial respiratory function. GMC and MTEC cells exposing in medium contain high glucose or TGF- $\beta$ displayed notably reduced basal and maximal respiration rates compared with that in low glucoses medium (Figure 8c-f). Treatment with 1907-B restored basal and maximal respiratory rates in high glucose or TGF- $\beta$ induced GMC and mTEC cells. Moreover, maximal respiratory capacity, and ATP turnover were found to be significantly reduced in high glucose or TGF- $\beta$-treated cells compared with the control group. However, 1907-B application improved the mitochondrial respiratory parameters in high glucose or TGF- $\beta$-treated cells. When PKA was inhibited, 1907-B was unable to increase OCR in GMC and mTEC cells, which indicated that the improved mitochondrial function was mediated by PKA signaling. These data demonstrated that 1907-B improved mitochondrial oxidative capacity directly via PKA signalling.

\section{Discussion}

The pathogenesis of CKD is complex. Many studies indicate that mitochondrial dysfunction, oxidative stress, inflammation, apoptosis and ECM deposition are implicated in the progression of renal diseases ${ }^{60}$. Therefore, strategies for CKD treatment should target a key factor(s) that is involved in prominent pathogenic pathways in this condition to ultimately inhibit multiple pathological features in a simultaneous manner ${ }^{5,61}$.

The present study, we identified GLP-1R and GCGR as key regulators of CKD, which were correlated with disease severity during progressive kidney disease in patients with CKD, as well as $\mathrm{db} / \mathrm{db}$ mice or UUO mice. Notably, downregulation of GLP-1R and GCGR in the kidney of $\mathrm{db} / \mathrm{db}$ mice significantly promoted more advanced diabetic nephropathy than wild-type mice, accompanied by increased renal fibrosis, inflammation and decreased mitochondrial function. Furthermore, immunofluorescence staining analyses demonstrated that GLP-1R was mainly expressed in the glomeruli and GCGR was expressed in whole kidney including renal tubules and glomeruli. Considering that the hypoglycemic effect and antioxidant effect of GLP-1 and the effect of glucagon on improving mitochondrial function, we speculate that targeting GLP-1R and GCGR could be considered as promising avenues for CKD treatment by preventing hyperglycemia and attenuating mitochondrial dysfunction.

On the basis of our findings, pharmacological approaches targeting GLP-1R and GCGR should ideally ameliorate mitochondrial function and block progression of renal injury. At present, the very large investment is put into the development of GLP-1R/GCGR co-agonists, some of them has been applied for the treatment of NASH, obesity and diabetes successfully. However, the effect of GLP-1R/GCGR coagonists on CKD treatment is still unknown ${ }^{62}$. In the present study, we have engineered GLP-1R/GCGR coagonist analogues that fulfill all the design criteria. Among them, we have set up to engineer a safe, highefficiency and ultralong-acting basal candidate, 1907-B (proteolytic stability, strong and reversible 
albumin binding, strong GLP-1/GCG receptor affinity). Stability toward proteolytic degradation was achieved by connecting C18 and "GGSGSG" with Lys in position 10 of 1907-B. Pharmacokinetic results in vivo showed that the half-life of 1907-B was prolonged 2-3-fold than semaglutide, which makes it available to administer twice a week or even less frequency.

Next, the improvement effect of 1907-B in DN was assessed in db/db mice. Indeed, 1907-B could significantly decrease the high blood glucose and ameliorate the renal injury. Interestingly, we found the GLP-1R-specific agonist semaglutide and GCGR-specific agonist glucagon also effectively inhibit renal damage, respectively. According to the study from Yamada et al. ${ }^{24}$, GLP-1R signaling has a crucial role in the development and progression of DN, and GLP-1R agonists such as exendin-4 and liraglutide attenuated diabetic renal injury through the protection of glomerular endothelial cells, reduction of renal oxidative stress and suppression of renal inflammatory cytokines, which could explain the pharmacologic action of semaglutide. On the other hand, despite glucagon caused higher blood glucose than control $\mathrm{db} / \mathrm{db}$ mice, it still obviously inhibited renal injury via modulating mitochondrial turnover, which indicated that mitochondrial function played a vital role in the process of DN and targeting glycolysis alone may not be a wise choice for DN treatment. Consistent with the selective pharmacological blockade studies reported here, the improvement effect of 1907-B on diabetic renal damage will be significantly affected in $\mathrm{db} / \mathrm{db}$ mice with down-regulated GLP-1R or GCGR expression. Moreover, it should be noted that 1907-B treatment still exhibited part anti-diabetic nephropathy effects in $\mathrm{db} / \mathrm{db}$ mice lacking GLP-1R, but not in $\mathrm{db} / \mathrm{db}$ mice lacking GCGR. Taken together, GCGR signaling has a more important role than GLP-1R signaling during 1907-B treatment for DN.

To explore whether 1907-B can also improve renal fibrosis, we performed the UUO surgery to build the renal fibrosis model and further investigated its renal phenotype under 1907-B treatment. Our results demonstrated that 1907-B treatment exhibited fewer tubular injuries and ECM deposition. Interestingly, glucagon had the most significant effect in improving renal fibrosis among these three treatment groups. Similarly, GLP-1R or GCGR knockdown assay also identified GCGR as a critical role in the treatment of 1907-B for renal fibrosis, which was supported by our findings that GCGR was localized in whole kidney including renal tubules and glomeruli and GLP-1R was mainly expressed in the glomeruli, whereas the primary feature of UUO was tubular injury.

Mechanistically, ample evidences have indicated that 1907-B could reduce renal injury via multiple mechanisms. 1907-B treatment attenuates renal oxidative stress and increases the expression of antioxidant factors via GLP-1 signaling. On the other hand, 1907-B could also improve mitochondrial turnover via glucagon signaling. In short, both of them can further improve mitochondrial function. Furthermore, 1907-B protects kidney against inflammation via NFKB pathway and renal fibrosis via TGF$\beta$-SMAD pathway. Finally, our data reveal that the treatment of 1907-B improve renal oxidative stress, inflammation, renal injury and fibrosis via targeting GLP-1R and GCGR, as summarized in Figure 9. From a clinical perspective, our study highly implicates the future development of a highly potent ultra-long acting and safe GLP-1R/GCGR co-agonist, 1907-B that disrupt the process of progressive renal disease as promising avenues for CKD therapy. Excitingly, the accredited institutions are conducting the 
preclinical studies of 1907-B in diabetic nephropathy, and the relevant results will be disclosed in the future.

\section{Methods}

Methods are available in the Supplementary Information.

\section{Data Availability}

Data supporting our findings in current study are available within this paper and its Supplementary Information files and from corresponding author on reasonable reasons. All the data supporting the findings of this study are available within this article, supplementary information files, and Source Data file (https://doi.org/10.6084/m9.figshare.17008105.v1). Source data are provided with this paper.

\section{Declarations}

\section{Acknowledgments}

We appreciate the financial support from the National Natural Science Foundation of China (No. 91853106), the Program for Guangdong Introducing Innovative and Enterpre-neurial Teams (No. 2016ZT06Y337), and the Fundamental Research Funds for the Central Universities (No. 19ykzd25). National Key Research and Development Program (No. 2017YFE0109900).

\section{Author contributions}

X.J. and R.W. conceived and designed the research and supervised the studies. G.C. provided the samples from patients. Q.Z. and J.D. performed the experiments and analyzed data. N.S. and H.X. supported the peptide synthesis and purification. S.Y. performed the cell experiments and the acquisition of data. H.C. and H.Y. performed the animal models. Z.L. construct the three-dimensional model of 1907/GLP-1R complex. S.Y. analyzed the pathological results from patients and finished the Remuzzi score. X.J. analyzed the data and wrote the manuscript.

\section{Competing financial interests}

The authors declare competing financial interests: details are available in the online version of the paper.

\section{References}

1. Zhao, Y.Y. Chronic kidney disease: Biomarker diagnosis to therapeutic targets. Clinica Chimica Acta 499, 54-63 (2019).

2. Ferenbach, D.A. \& Bonventre, J.V. Acute kidney injury and chronic kidney disease: From the laboratory to the clinic. Néphrologie \& Thérapeutique, S41-S48 (2016). 
3. Nahas, A., Muchaneta-Kubara, E.C., Essaway, M. \& Soylemezoglu, O. Renal fibrosis: insights into pathogenesis and treatment. Int J Biochem Cell Biol 29, 55-62 (1997).

4. Liu, Y., Wang, Y., Ding, W. \& Wang, Y. Mito-TEMPO alleviates renal fibrosis by reducing inflammation, mitochondrial dysfunction, and endoplasmic reticulum stress. Oxid Med Cell Longev 2018, 5828120 (2018).

5. Nastase, M.V., Zeng-Brouwers, J., Wygrecka, M. \& Schaefer, L. Targeting renal fibrosis: Mechanisms and drug delivery systems. Adv Drug Deliv Rev 129, 295-307 (2018).

6. Delles, C. \& Vanholder, R. Chronic kidney disease. Clin Sci (Lond) 131, 225-226 (2017).

7. D Uni, A., Liakopoulos, V., Roumeliotis, S., Peschos, D. \& Dounousi, E. Oxidative Stress in the Pathogenesis and Evolution of Chronic Kidney Disease: Untangling Ariadne's Thread. Int J Mol Sci 20, 3711 (2019).

8. Ruiz, S., Pergola, P.E., Zager, R.A. \& Vaziri, N.D. Targeting the transcription factor Nrf2 to ameliorate oxidative stress and inflammation in chronic kidney disease. Kidney Int 83, 1029-1041 (2013).

9. Hu, R., Wang, M.Q., Liu, L.Y., You, H.Y., Wu, X.H., Liu, Y.Y., Wang, Y.J., Lu, L., Xiao, W., Wei, L.B. Calycosin inhibited autophagy and oxidative stress in chronic kidney disease skeletal muscle atrophy by regulating AMPK/SKP2/CARM1 signalling pathway. J Cell Mol Med 24, 11084-11099 (2020).

10. Sureshbabu, A., Ryter, S.W. \& Choi, M.E. Oxidative stress and autophagy: crucial modulators of kidney injury. Redox Biol 4, 208-214 (2015).

11. Rapa, S.F., Iorio, B., Campiglia, P., Heidland, A. \& Marzocco, S. Inflammation and oxidative stress in chronic kidney disease-potential therapeutic role of minerals, vitamins and plant-derived metabolites. Int J Mol Sci 21, 263 (2020).

12. Moreno, J.A., et al. Targeting inflammation in diabetic nephropathy: a tale of hope. Expert opinion on investigational drugs 27, 917-930 (2018).

13. Czaya, B. \& Faul, C. FGF23 and inflammation-a vicious coalition in CKD. Kidney Int 96, 813-815 (2019).

14. Chen, $\mathrm{H}$., et al. RIPK3-MLKL-mediated necroinflammation contributes to AKI progression to CKD. Cell death \& disease 9,878 (2018).

15. Nogueira, A., Pires, M.J. \& Oliveira, P.A. Pathophysiological Mechanisms of Renal Fibrosis: A Review of Animal Models and Therapeutic Strategies. In Vivo 31, 1-22 (2017).

16. Bhargava, P. \& Schnellmann, R.G. Mitochondrial energetics in the kidney. Nat Rev Nephro/ 13, 629646 (2017).

17. Pieczenik, S.R. \& Neustadt, J. Mitochondrial dysfunction and molecular pathways of disease. Exp Mol Pathol 83, 84-92 (2007).

18. Che, R., Yuan, Y., Huang, S. \& Zhang, A. Mitochondrial dysfunction in the pathophysiology of renal diseases. Am J Physiol Renal Physio/ 306, 367-378 (2014). 
19. Elmarakby, A.A. \& Sullivan, J.C. Relationship between oxidative stress and inflammatory cytokines in diabetic nephropathy. Cardiovasc Ther 30, 49-59 (2012).

20. Bai, M., et al. MicroRNA-214 promotes chronic kidney disease by disrupting mitochondrial oxidative phosphorylation. Kidney Int 95, 1389-1404 (2019).

21. Small, D.M., Coombes, J.S., Bennett, N., Johnson, D.W. \& Gobe, G.C. Oxidative stress, anti-oxidant therapies and chronic kidney disease. Nephrology 17, 311-321 (2012).

22. Duann, P. \& Lin, P.H. Mitochondria Damage and Kidney Disease. Adv Exp Med Bio/ 982, 529-551 (2017).

23. Jha, J.C., et al. Genetic targeting or pharmacologic inhibition of NADPH oxidase nox4 provides renoprotection in long-term diabetic nephropathy. J Am Soc Nephrol 25, 1237-1254 (2014).

24. Fujita, H., et al. The protective roles of GLP-1R signaling in diabetic nephropathy: possible mechanism and therapeutic potential. Kidney Int 85, 579-589 (2014).

25. Drucker, D.J. Mechanisms of Action and Therapeutic Application of Glucagon-like Peptide-1. Cell Metab 27, 740-756 (2018).

26. Farah, L.X., et al. The physiological role of glucagon-like peptide-1 in the regulation of renal function. Am J Physiol Renal Physio/ 310, F123-127 (2016).

27. Thomas, M.C. The potential and pitfalls of GLP-1 receptor agonists for renal protection in type 2 diabetes. Diabetes Metab 43, 2S20-22S27 (2017).

28. Boland, M.L., et al. Resolution of NASH and hepatic fibrosis by the GLP-1R and GCGR dual-agonist cotadutide via modulating mitochondrial function and lipogenesis. Nat Metab 2, 413-431 (2020).

29. Gault, V.A., Bhat, V.K., Irwin, N. \& Flatt, P.R. A novel glucagon-like peptide-1 (GLP-1)/glucagon hybrid peptide with triple-acting agonist activity at glucose-dependent insulinotropic polypeptide, GLP-1, and glucagon receptors and therapeutic potential in high fat-fed mice. J Biol Chem 288, 35581-35591 (2013).

30. Kannt, A., et al. Incretin combination therapy for the treatment of non-alcoholic steatohepatitis. Diabetes Obes Metab 22, 1328-1338 (2020).

31. Day, J.W., et al. A new glucagon and GLP-1 co-agonist eliminates obesity in rodents. Nat Chem Bio/ 5, 749-757 (2009).

32. Clemmensen C, C.J., Finan B, Sullivan L, Fischer K, Küchler D, Sehrer L, Ograjsek T, Hofmann SM, Schriever SC, Pfluger PT, Pinkstaff J, Tschöp MH, Dimarchi R, Müller TD. GLP-1/Glucagon Coagonism Restores Leptin Responsiveness in Obese Mice Chronically Maintained on an Obesogenic Diet. Diabetes Obes Metab 63, 1422-1427 (2014).

33. Pocai, A., et al. Glucagon-like peptide $1 /$ glucagon receptor dual agonism reverses obesity in mice. Diabetes 58, 2258-2266 (2009).

34. Day, J.W., et al. Optimization of co-agonism at GLP-1 and glucagon receptors to safely maximize weight reduction in DIO-rodents. Biopolymers 98, 443-450 (2012). 
35. Finan B, M.T., Ottaway N, Müller TD, Habegger KM, Heppner KM, Kirchner H, Holland J, Hembree J, Raver C, Lockie SH, Smiley DL, Gelfanov V, Yang B, Hofmann S, Bruemmer D, Drucker DJ, Pfluger PT, Perez-Tilve D, Gidda J, Vignati L, Zhang L, Hauptman JB, Lau M, Brecheisen M, Uhles S, Riboulet W, Hainaut E, Sebokova E, Conde-Knape K, Konkar A, DiMarchi RD, Tschöp MH. Unimolecular dual incretins maximize metabolic benefits in rodents, monkeys, and humans. Sci Trans/ Med 5, 209ra151 (2013).

36. Finan, B., et al. A rationally designed monomeric peptide triagonist corrects obesity and diabetes in rodents. Nat Med 21, 27-36 (2015).

37. Pocai, A. Action and therapeutic potential of oxyntomodulin. Mol Metab 3, 241-251 (2014).

38. Philip, A., et al. MEDI0382, a GLP-1 and glucagon receptor dual agonist, in obese or overweight patients with type 2 diabetes: a randomised, controlled, double-blind, ascending dose and phase $2 \mathrm{a}$ study. The Lancet 391, 2607-2618 (2018).

39. Glessner, M. American Diabetes Association - 75th Scientific Sessions (June 5-9, 2015 - Boston, Massachusetts, USA). Drugs Today (Barc) 51, 383-386 (2015).

40. Wang, Y., et al. Multifunctional antibody agonists targeting glucagon-like peptide-1, glucagon, and glucose-dependent insulinotropic polypeptide receptors. Angew Chem Int Ed Eng/ 55, 12475-12478 (2016).

41. Yang, P.Y., et al. Engineering a long-acting, potent GLP-1 analog for microstructure-based transdermal delivery. Proc Natl Acad Sci U S A 113, 4140-4145 (2016).

42. Muppidi, A., et al. Design of Potent and Proteolytically Stable Oxyntomodulin Analogs. ACS Chem Biol 11, 324-328 (2016).

43. Ma, T., et al. A novel long-acting oxyntomodulin analogue eliminates diabetes and obesity in mice. Eur J Med Chem 203, 112496 (2020).

44. Kjeldsen, T.B., et al. Engineering of Orally Available, Ultralong-Acting Insulin Analogues: Discovery of OI338 and 01320. J Med Chem 64, 616-628 (2021).

45. Wang, W., et al. DR10601, a novel recombinant long-acting dual glucagon-like peptide-1 and glucagon receptor agonist for the treatment of obesity and type 2 diabetes mellitus. J Endocrinol Invest 43, 653-662 (2020).

46. Pratley, et al. Semaglutide versus dulaglutide once weekly in patients with type 2 diabetes (SUSTAIN 7): a randomised, open-label, phase 3b trial. Lancet Diabetes Endocrinol 6, 275-286 (2018).

47. Lau, J., et al. Discovery of the once-weekly glucagon-like peptide-1 (GLP-1) analogue semaglutide. $J$ Med Chem 58, 7370-7380 (2015).

48. Kulkarni, T.A., et al. A year-long extended release nanoformulated cabotegravir prodrug. Nat Mater 19, 910-920 (2020).

49. Schievenbusch S, S.I., Scheffler M, Nischt R, Coutelle O, Hösel M, Hallek M, Fries JW, Dienes HP, Odenthal $\mathrm{M}$, Büning $\mathrm{H}$. Combined paracrine and endocrine AAV9 mediated expression of hepatocyte growth factor for the treatment of renal fibrosis. Mol Ther 18, 1302-1309 (2010). 
50. Jiang, N., et al. Design of novel Xenopus GLP-1-based dual glucagon-like peptide 1 (GLP-1)/glucagon receptor agonists. Eur J Med Chem 212, 113118 (2021).

51. Lee, E.S. \& Youn, Y.S. Albumin-based potential drugs: focus on half-life extension and nanoparticle preparation. J Pharm Investig 46, 305-315 (2016).

52. Giralt-López, A., et al. Revisiting Experimental Models of Diabetic Nephropathy. Int J Mol Sci 21(2020).

53. Yiu, W.H., Lin, M. \& Tang, S.C. Toll-like receptor activation: from renal inflammation to fibrosis. Kidney international supplements 4, 20-25 (2014).

54. Li, A., et al. LincRNA 1700020114Rik alleviates cell proliferation and fibrosis in diabetic nephropathy via miR-34a-5p/Sirt1/HIF-1a signaling. Cell death \& disease 9, 461 (2018).

55. Rigalleau, V., et al. Normoalbuminuric renal-insufficient diabetic patients: a lower-risk group. Diabetes care 30, 2034-2039 (2007).

56. Gao, P., et al. Yin Yang 1 protein ameliorates diabetic nephropathy pathology through transcriptional repression of TGFß1. Sci Transl Med 11(2019).

57. Bai, Y., et al. Ruxolitinib alleviates renal interstitial fibrosis in UUO mice. Int J Biol Sci 16, 194-203 (2020).

58. Zhao, X., Kwan, J.Y.Y., Yip, K., Liu, P.P. \& Liu, F.F. Targeting metabolic dysregulation for fibrosis therapy. Nat Rev Drug Discov 19, 57-75 (2020).

59. Du, K., Ramachandran, A. \& Jaeschke, H. Oxidative stress during acetaminophen hepatotoxicity: Sources, pathophysiological role and therapeutic potential. Redox Biology 10, 148-156 (2013).

60. Wei, D., Guo, H., Xu, C., Wang, B. \& Feng, D. Mitochondrial reactive oxygen species-mediated NLRP3 inflammasome activation contributes to aldosterone-induced renal tubular cells injury. Oncotarget 7 , 17479-17491 (2016).

61. Bhattacharjee, N., Barma, S., Konwar, N., Dewanjee, S. \& Manna, P. Mechanistic insight of diabetic nephropathy and its pharmacotherapeutic targets: An update. Eur J Pharmacol 791, 8-24 (2016).

62. Cui, J., Shang, A., Wang, W. \& Chen, W. Rational design of a GLP-1/GIP/Gcg receptor triagonist to correct hyperglycemia, obesity and diabetic nephropathy in rodent animals. Life Sci 260, 118339 (2020).

\section{Figures}


a

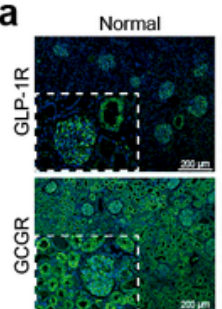

C
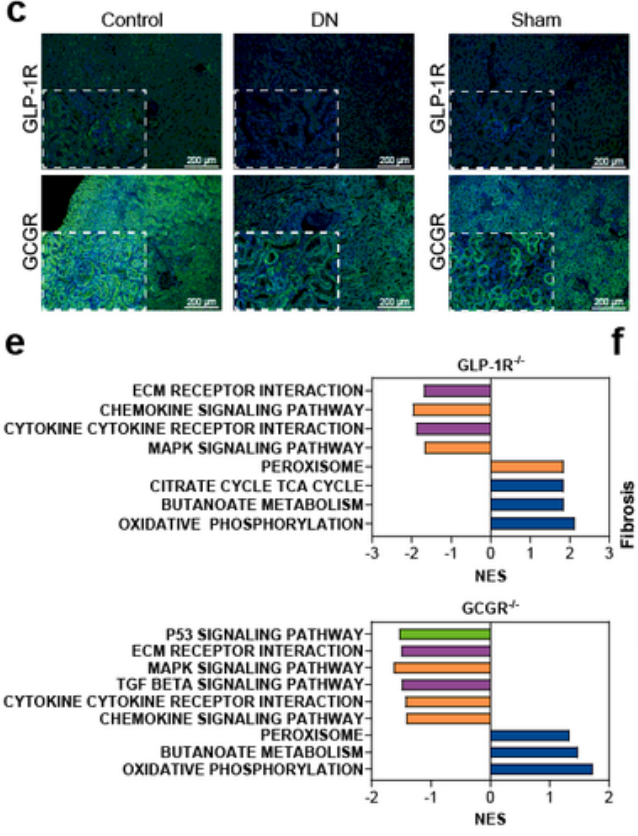

b

Normal

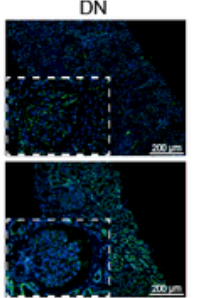

Sham
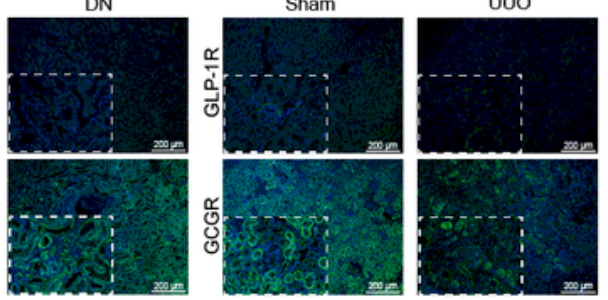

f

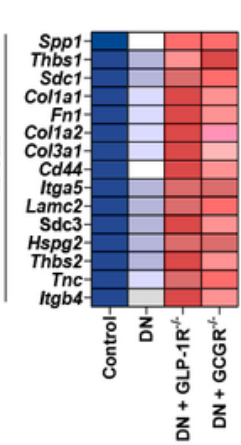

d
CKD

$\mathrm{DN}$
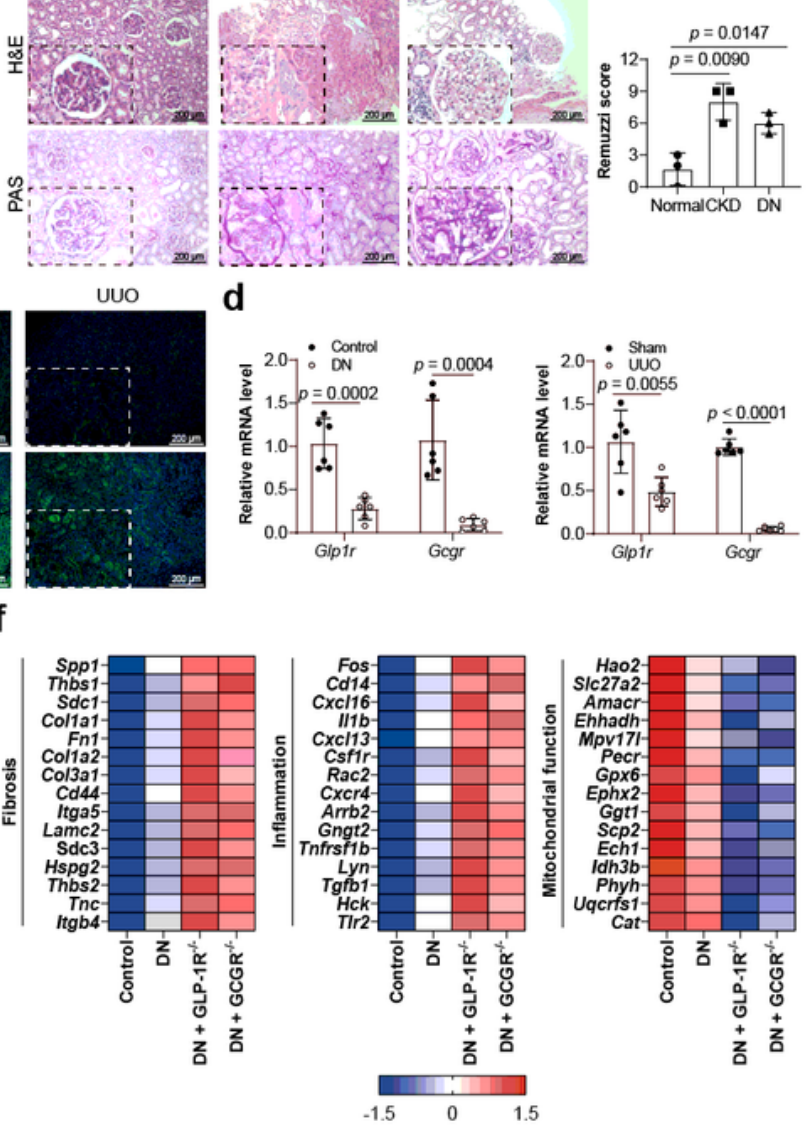

\section{Figure 1}

GLP-1R and GCGR expression is correlated with progressive renal disease. a, GLP-1R and GCGR immunofluorescence staining of kidneys from patients with diabetic nephropathy or other chronic kidney disease. b, H\&E, PAS staining and Remuzzi score of kidneys from patients with diabetic nephropathy or other chronic kidney disease. c, GLP-1R and GCGR immunofluorescence staining of kidneys from db/db or UUO mice. d, The mRNA levels of Glp1rand Gcgr expression in kidneys from db/db or UUO mice. e, 
GSEA of pathways related to fibrosis, inflammation and mitochondrial function in $\mathrm{db} / \mathrm{db}$ mice treated with AAV9-CON, AAV9-GLP1R-RNAi or AAV9-GCGR-RNAi. f, Heatmap of fibrosis-, inflammation- and mitochondrial function-related gene expression profiles based on the RNA-Seq dataset. Here and later, unless otherwise indicated, for each assay, three separated experiments were performed. And for each experiment ( $n=3$ cell samples/group or $n=3-6$ mice/group). All the above data are presented as mean values \pm SEM using unpaired Student's $t$ test. Source data are provided as a Source Data file.

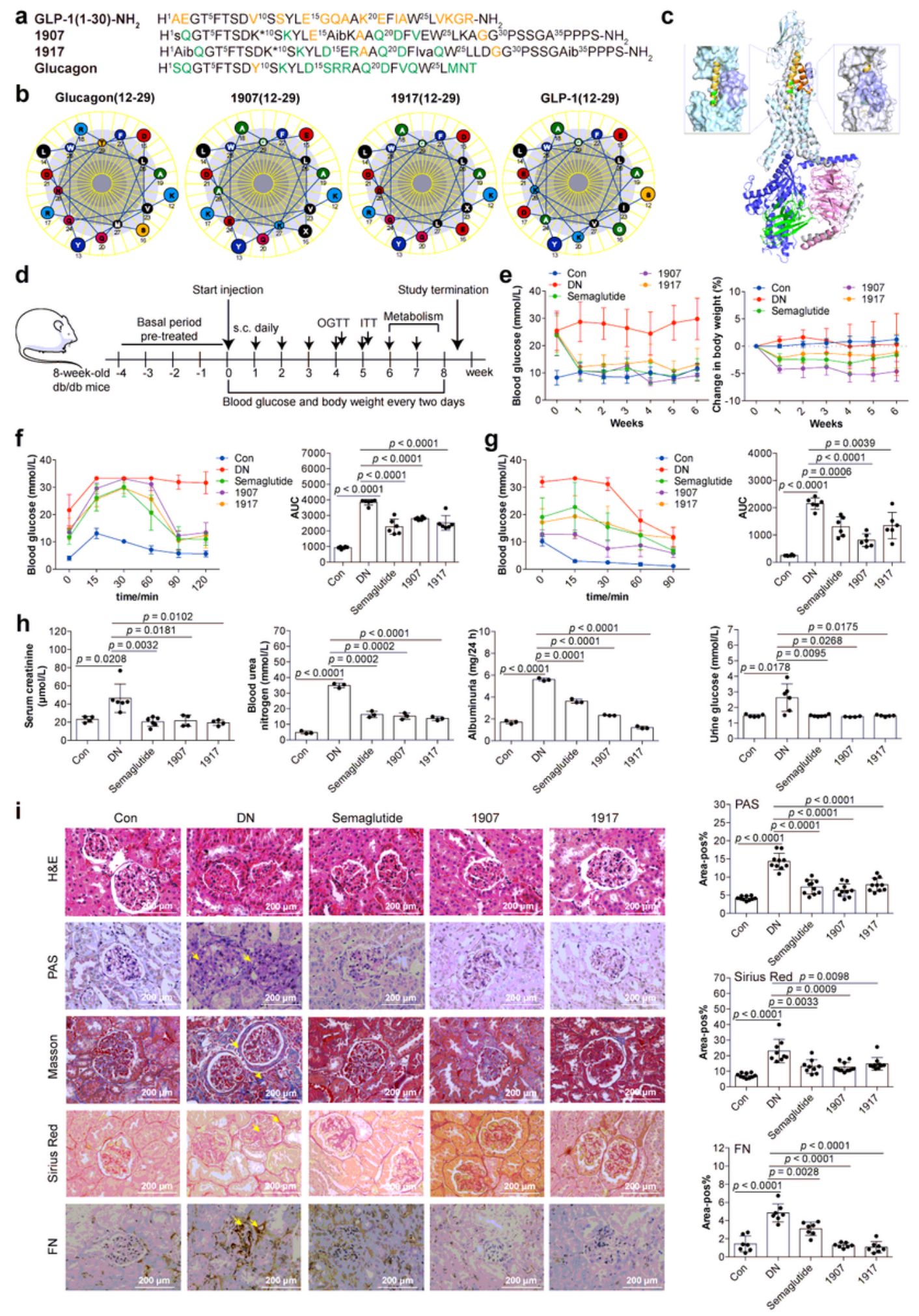




\section{Figure 2}

1907 generates synergistic metabolic benefits and ameliorates renal injury in $\mathrm{db} / \mathrm{db}$ mice. a, Sequence information of GLP-1, glucagon, 1907 and 1917. b, Helical wheel representation of GLP-1, glucagon, 1907 and 1917. c, Three- dimensional model of 1907/GLP-1R (PDB ID: 7LCJ).d, Schematic of the experimental procedure to test the pharmacological effects of 1907 and 1917 on $\mathrm{db} / \mathrm{db}$ mice. e, The fasting blood glucose and body weight change of $\mathrm{db} / \mathrm{db}$ mice after 6-week administration with 1907 or 1917 . $\mathrm{f}-\mathrm{g}$, Oral glucose tolerance test (OGTT) and insulin tolerance test (ITT) were performed at the 4 th or 5 th week of 1907 or 1917 administration, respectively. The corresponding area under curves (AUC) of blood glucose levels were calculated. $\mathrm{h}$, Serum creatinine, blood urea nitrogen, $24 \mathrm{~h}$-albuminuria in urine and urine glucose levels of $\mathrm{db} / \mathrm{db}$ mice at study termination. i, Representative images of H\&E, PAS, Masson and Sirius Red staining and immunohistochemistry staining of FN on kidneys from $\mathrm{db} / \mathrm{db}$ mice at indicated groups and statistical positive area, respectively. 


\section{a}

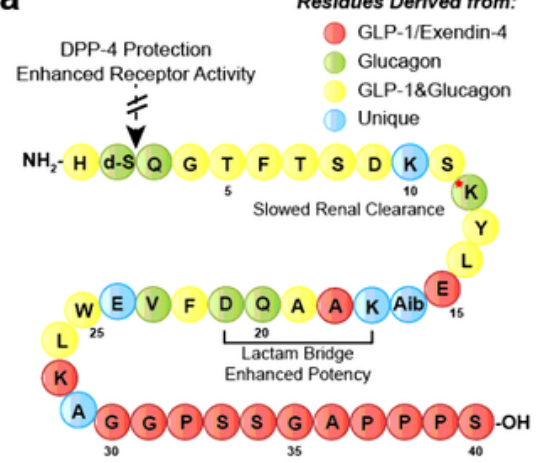

b ${ }^{*}$ K: Linkers and protractors

Single-site recognition

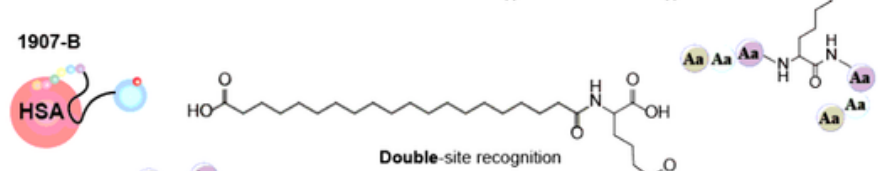

Aa Aa AB
C
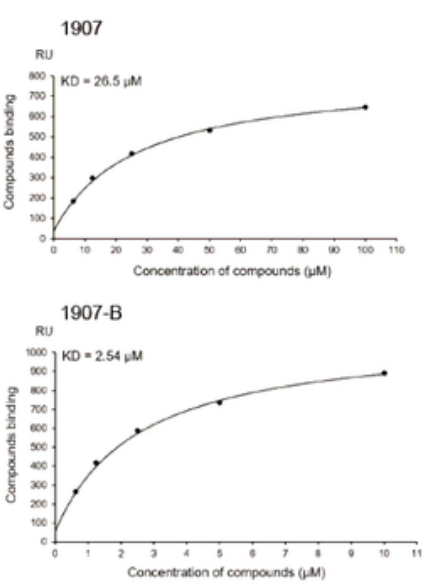

d
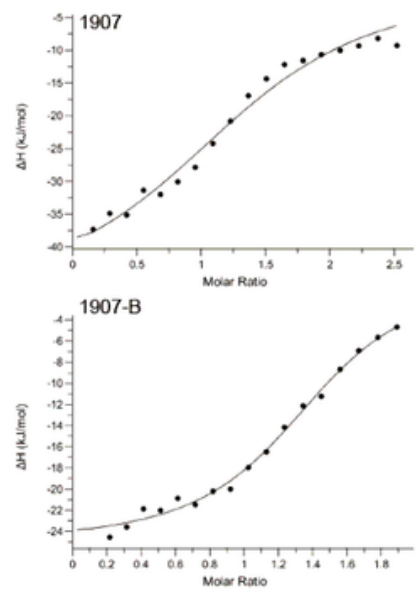
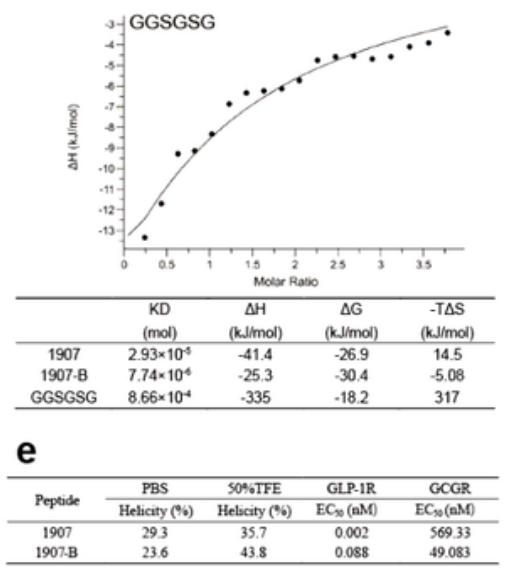
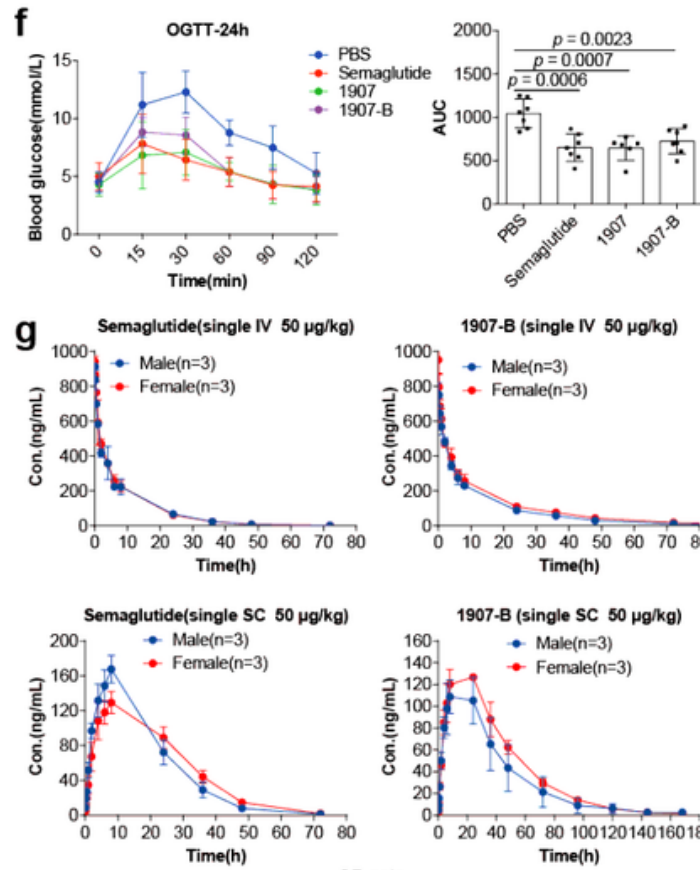

SD rats
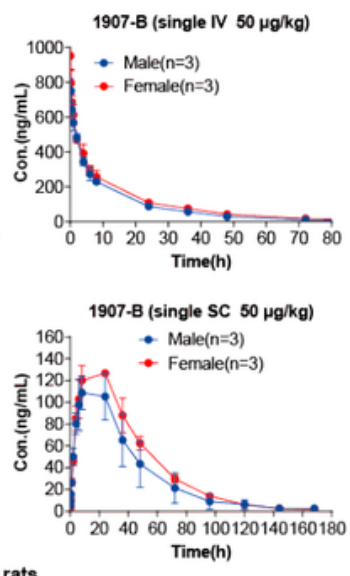
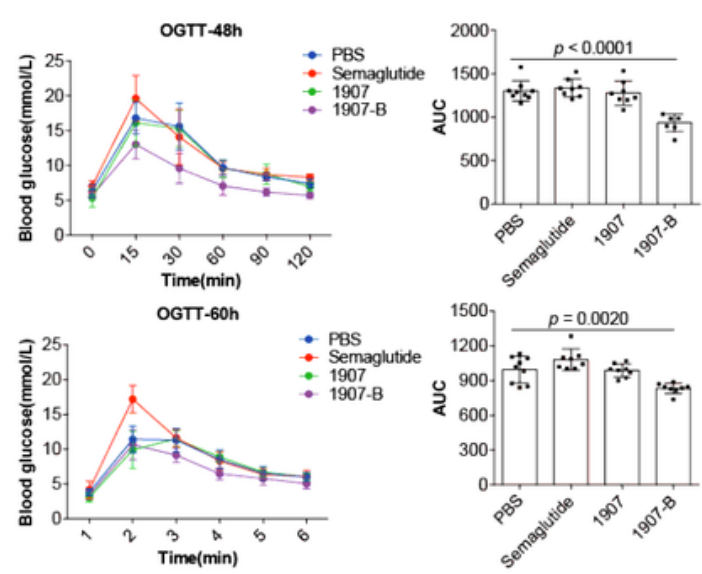

h $\quad 1907-8$ (single IV 25ug/kg)

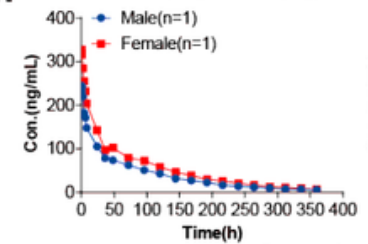

Cynomolgus monkeys

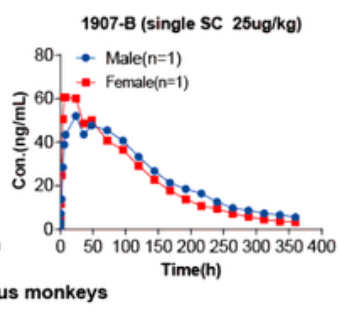

\section{Figure 3}

A rationally designed 1907 analogue, 1907-B, exhibits an extended half-life in vivo. a, The modification strategy to extend the half-life of 1907. b, Chemical structures of the linkers and protractors of 1907 or 1907-B. c, Surface plasmon resonance (SPR) sensorgrams showing binding of 1907 and 1907-B to human serum albumin. $\mathbf{d}$, Representative thermograms from isothermal titration calorimetry (ITC) measurements for the interaction of human serum albumin with 1907, 1907-B and GGSGSG. e, The 
calculated helicity of 1907 or 1907-B in PBS or 50\% TFE and in vitro activity of 1907 or 1907-B in the GLP-1R- or GCGR-mediated CRE-Luc reporter assay. $\mathbf{f}$, Oral glucose tolerance test (OGTT) was performed at the $24 \mathrm{~h}, 48 \mathrm{~h}$ and $60 \mathrm{~h}$ after peptides administration, respectively. The corresponding area under curves (AUC) of blood glucose levels were calculated. g, Pharmacokinetics of semaglutide or 1907-B in SD rats. h, Pharmacokinetics of 1907-B in cynomolgus monkeys.

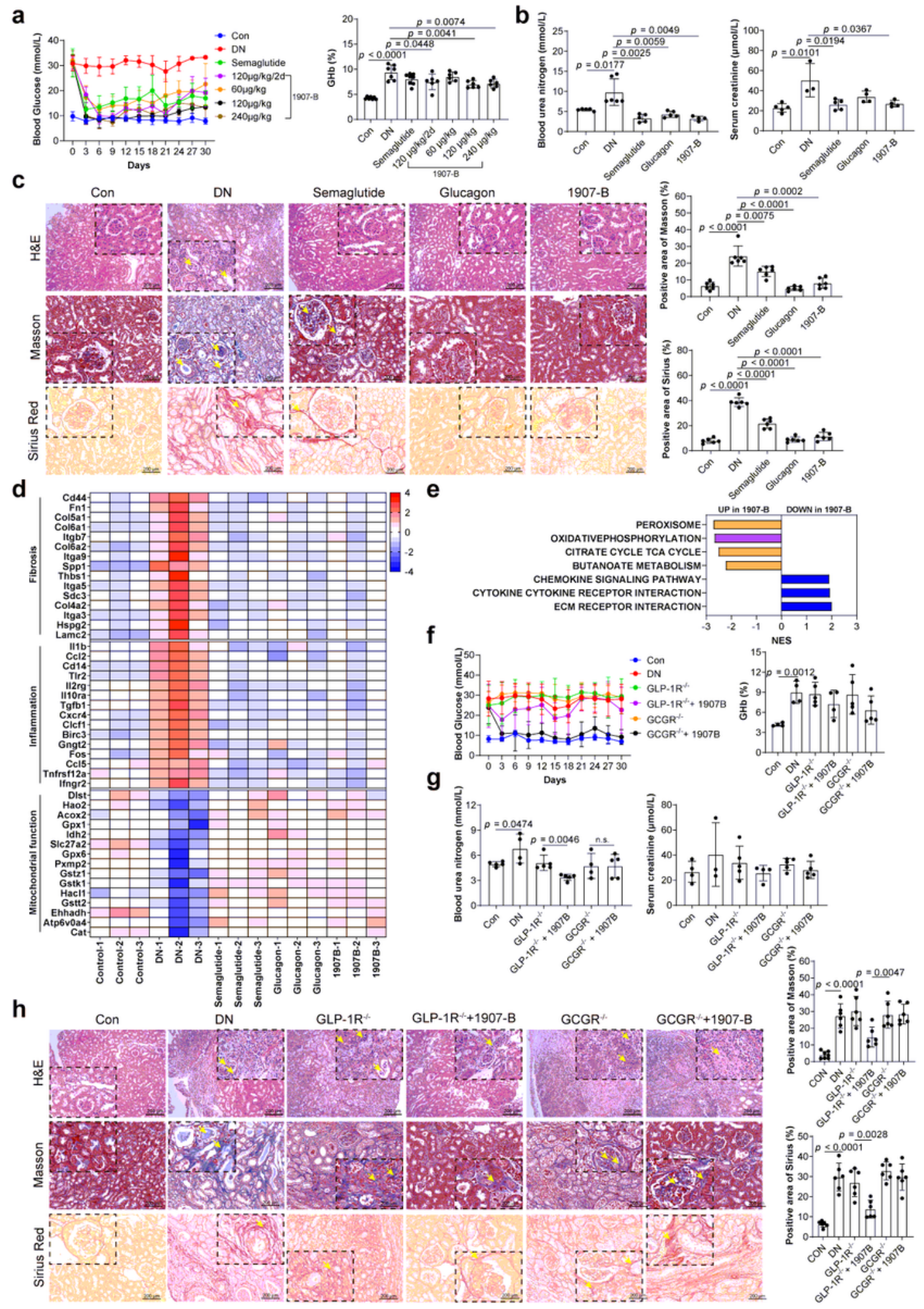




\section{Figure 4}

1907-B improves renal injury depend on GLP-1R and GCGR signaling in db/db mice. a, The fasting blood glucose and glycated hemoglobin of db/db mice after 4-week administration with semaglutide or 1917-B. b, Blood urea nitrogen and serum creatinine levels of $\mathrm{db} / \mathrm{db}$ mice treated with semaglutide, glucagon or 1907-B at study termination. c, Representative images of H\&E, Masson and Sirius Red staining on kidneys from $\mathrm{db} / \mathrm{db}$ mice at indicated groups and statistical positive area, respectively. $\mathrm{d}$, Heatmap of fibrosis-, inflammation- and mitochondrial function-related gene expression profiles based on the RNASeq dataset. e, GSEA of pathways related to fibrosis, inflammation and mitochondrial function in $\mathrm{db} / \mathrm{db}$ mice treated with 1907-B. f, The fasting blood glucose and glycated hemoglobin of GLP-1R $\mathrm{R}^{-/}$or GCGR $\mathrm{GC}^{-/}$ $\mathrm{db} / \mathrm{db}$ mice after-4 week administration with 1917-B. g, Blood urea nitrogen and serum creatinine levels of GLP-1R $\mathrm{R}^{-/}$or $\mathrm{GCGR}^{-/} \mathrm{db} / \mathrm{db}$ mice treated with 1907-B at study termination. $h$, Representative images of $\mathrm{H} \& \mathrm{E}$, Masson and Sirius Red staining on kidneys from GLP-1R $\mathrm{R}^{-/-}$or $\mathrm{GCGR}^{-/-} \mathrm{db} / \mathrm{db}$ mice at indicated groups and statistical positive area, respectively. 


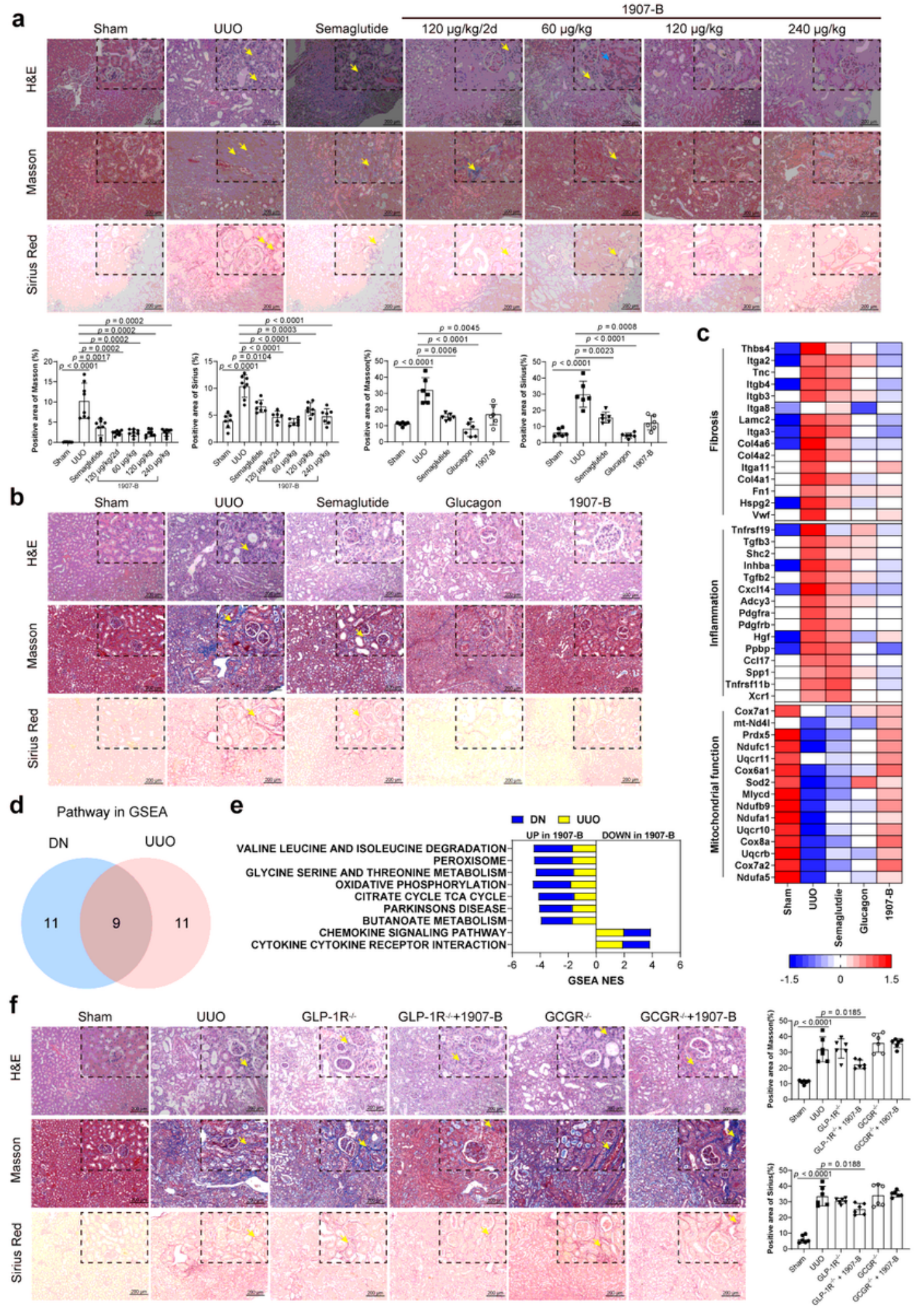

Figure 5

1907-B improves renal fibrosis depend on GLP-1R and GCGR signaling in UUO mice. a, Representative images of H\&E, Masson and Sirius Red staining on kidneys from UUO mice treated with semaglutide or 1907-B at different dose and statistical positive area, respectively. b, Representative images of H\&E, Masson and Sirius Red staining on kidneys from UUO mice treated with semaglutide, glucagon or 1907-B and statistical positive area, respectively. c, Heatmap of fibrosis-, inflammation- and mitochondrial 
function-related gene expression profiles based on the RNA-Seq dataset. de, Venn diagram and the corresponding scores of the intersecting pathways based on the transcriptomic data from $\mathrm{db} / \mathrm{db}$ mouse and UUO mouse kidney samples. f, Representative images of H\&E, Masson and Sirius Red staining on kidneys from GLP-1R $\mathrm{R}^{-/-}$or GCGR $\mathrm{GCO}^{-/-}$UU mice treated with 1907-B and statistical positive area, respectively.

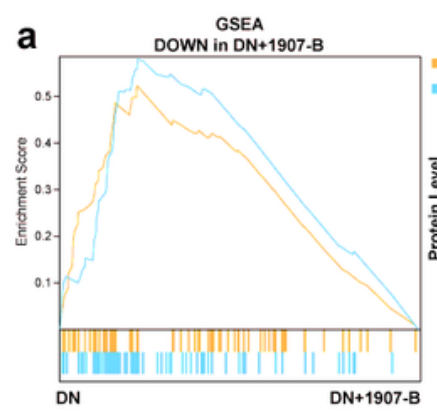

C
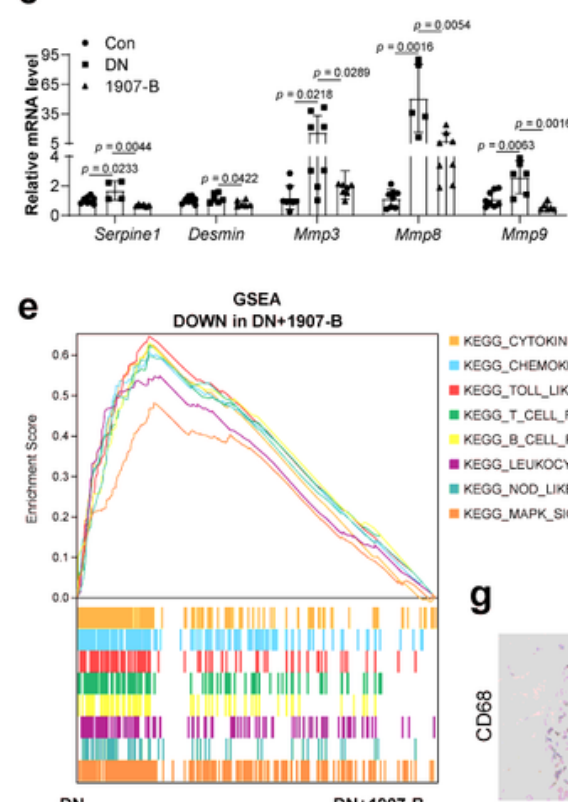

\section{(6)}

g

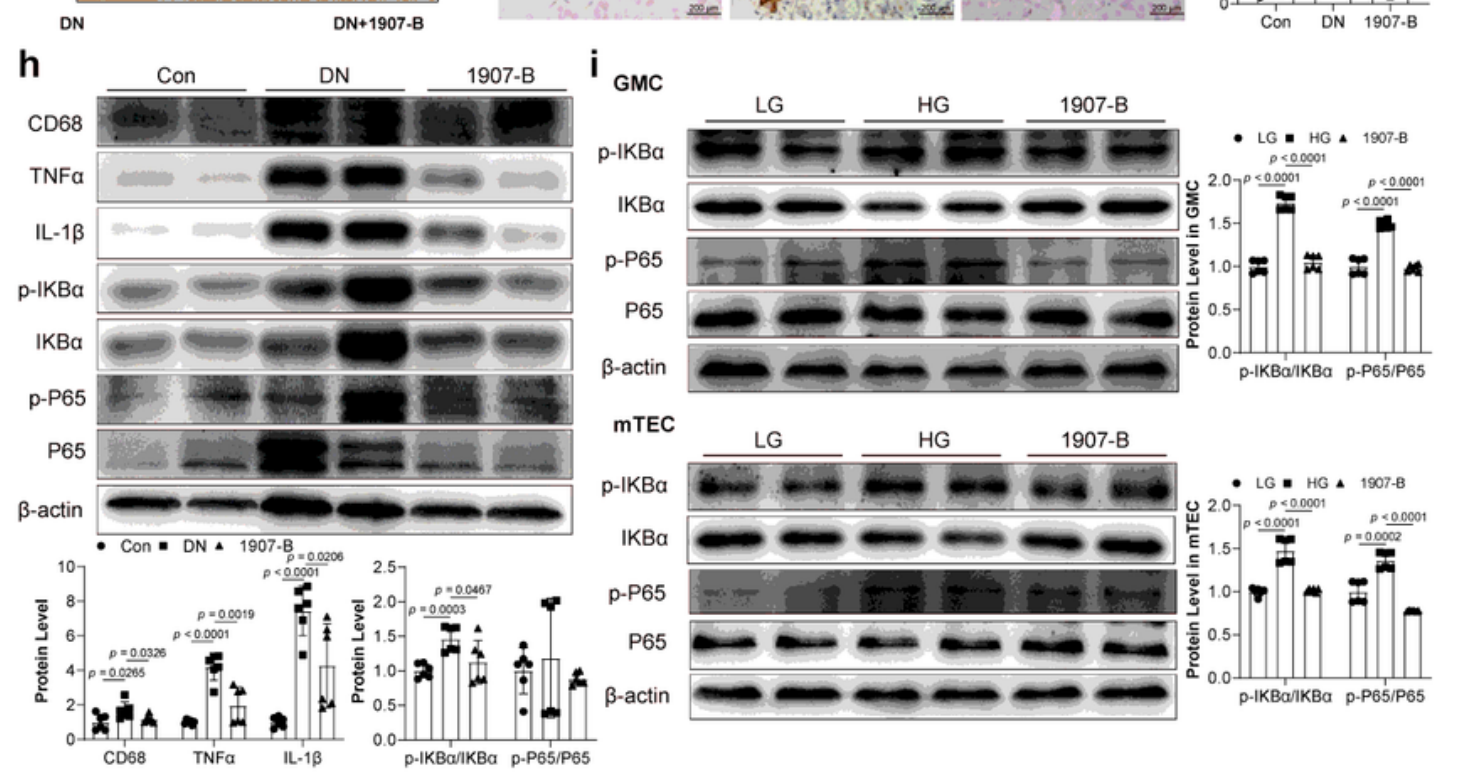

b $=$ KEGG_VEGF_SIGNALING_PATHWAY
$=$ KEGG_ECM_RECEPTOR_INTERACTION
- CON - DN 4 1907-B
$p<0.0001$

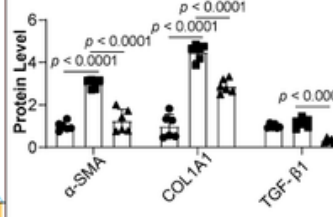

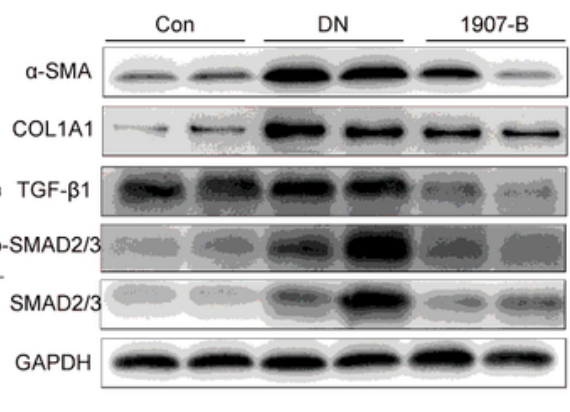

d GMC
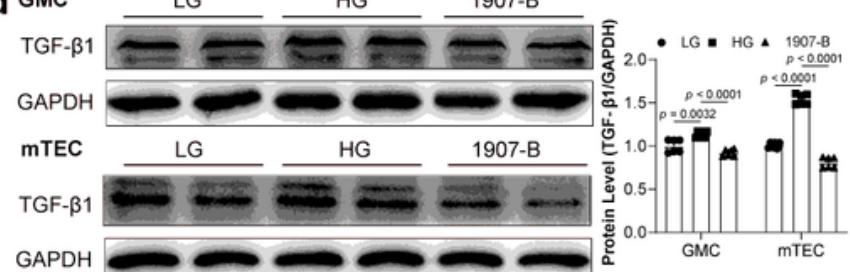

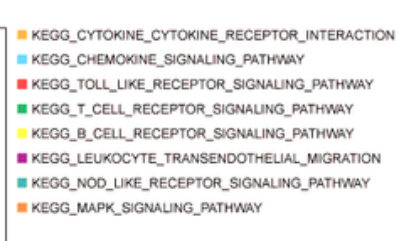
f
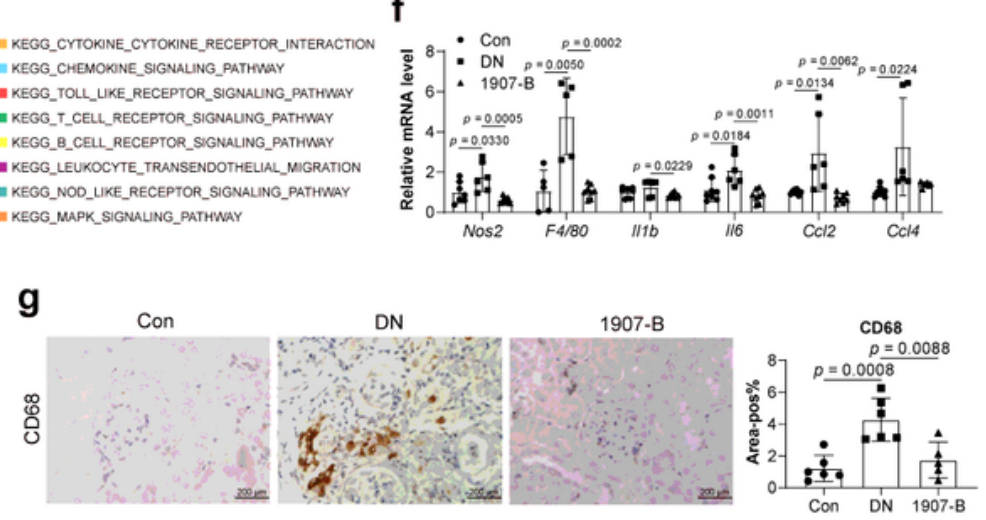


\section{Figure 6}

\section{7-B ameliorates renal inflammation and fibrosis via IKBa-NF-KB and TGF- $\beta 1$-Smad signaling}

pathways. a, GSEA pathway enrichment analysis of pathways related to fibrosis. $\mathbf{b}$, The expression level of key protein a-SMA, COL1A1, TGF- $\beta 1$ and p-SMAD2/3/SMAD2/3 in the kidney of the indicated groups. Here and later, unless otherwise indicated, the quantifications of protein expression level were performed using three independent western blotting experiments. c, the mRNA level of Serpine1, Desmin, Mmp3, $M m p 8$, and $M m p 9$ were measured in the kidney samples of the indicated groups. $\mathbf{d}$, The protein expression level of TGF- $\beta 1$ in GMC and mTEC cells of the indicated groups. e, GSEA pathway enrichment analysis of pathways related to inflammation. $f$, the mRNA level of Nos2, F4/80, $I / 1 \mathrm{~b}, \| / 6, C c / 2$ and $C c / 4$ were measured in the kidney samples of the indicated groups. $\mathbf{g}$, Representative images of immunohistochemistry staining of CD68 on kidneys from $\mathrm{db} / \mathrm{db}$ mice at indicated groups and statistical positive area, respectively. $\mathbf{h}$, The expression level of key protein CD68, TNFa, IL-1 $\beta, p-I K B a / I K B a$ and $p$ P65/P65 in the kidney of the indicated groups. $\mathbf{i}$, The protein expression level of $p$-IKBa/IKBa and $p$ P65/P65 in GMC and mTEC cells of the indicated groups. 


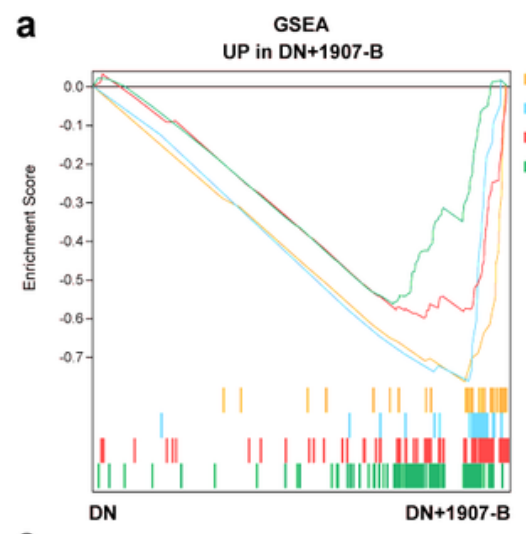

$=$ KEGG_BUTANOATE_METABOLISM
$=$ KEGG_CITRATE_CYCLE_TCA_CYCLE
$=$ KEGG_PEROXISOME
$=$ KEGG_OXIDATIVE_PHOSPHORYLATION

C
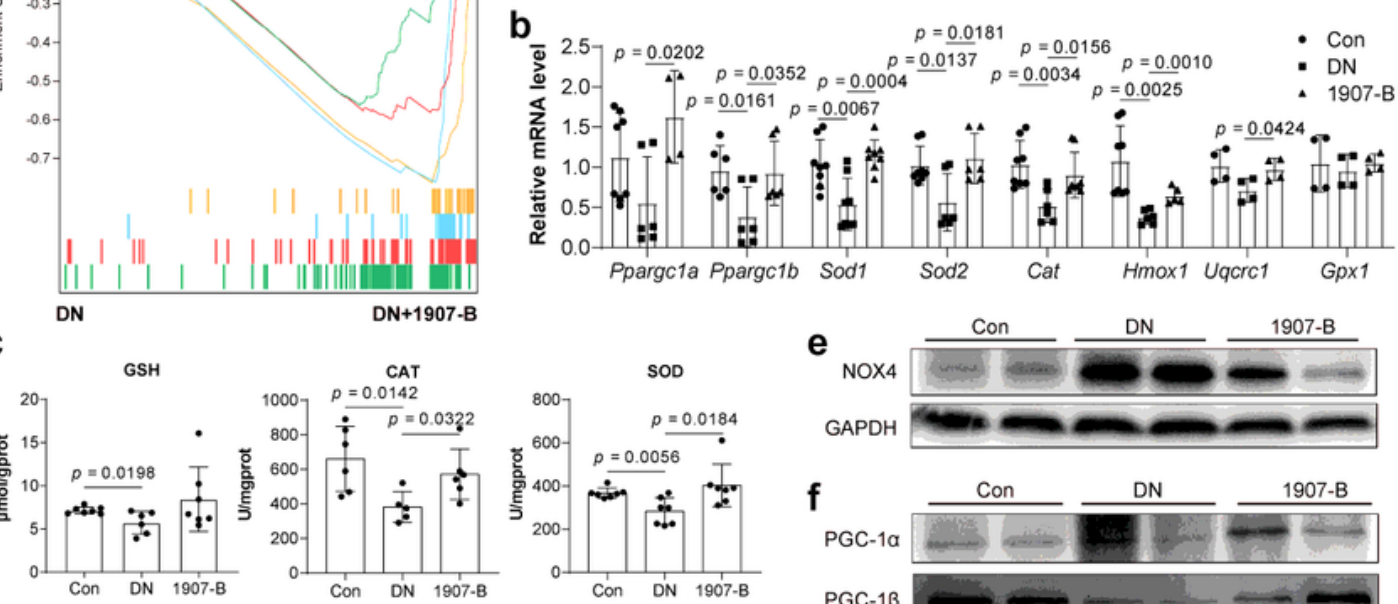

d

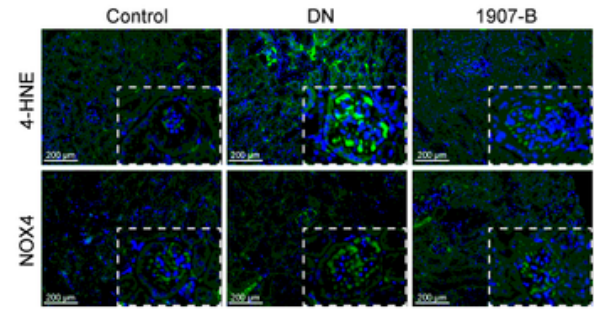

g

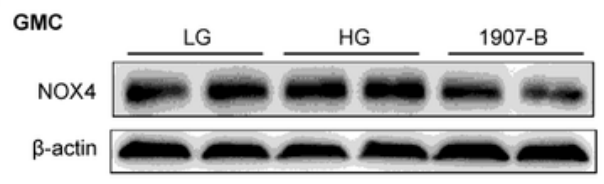

h

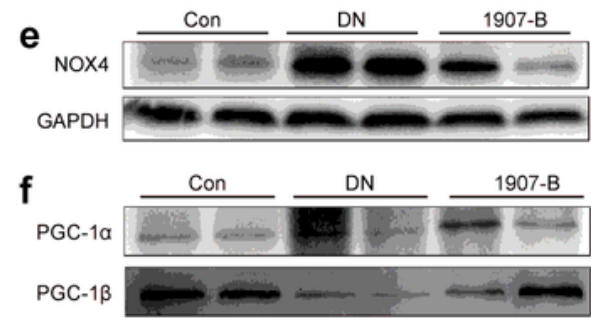

mTEC
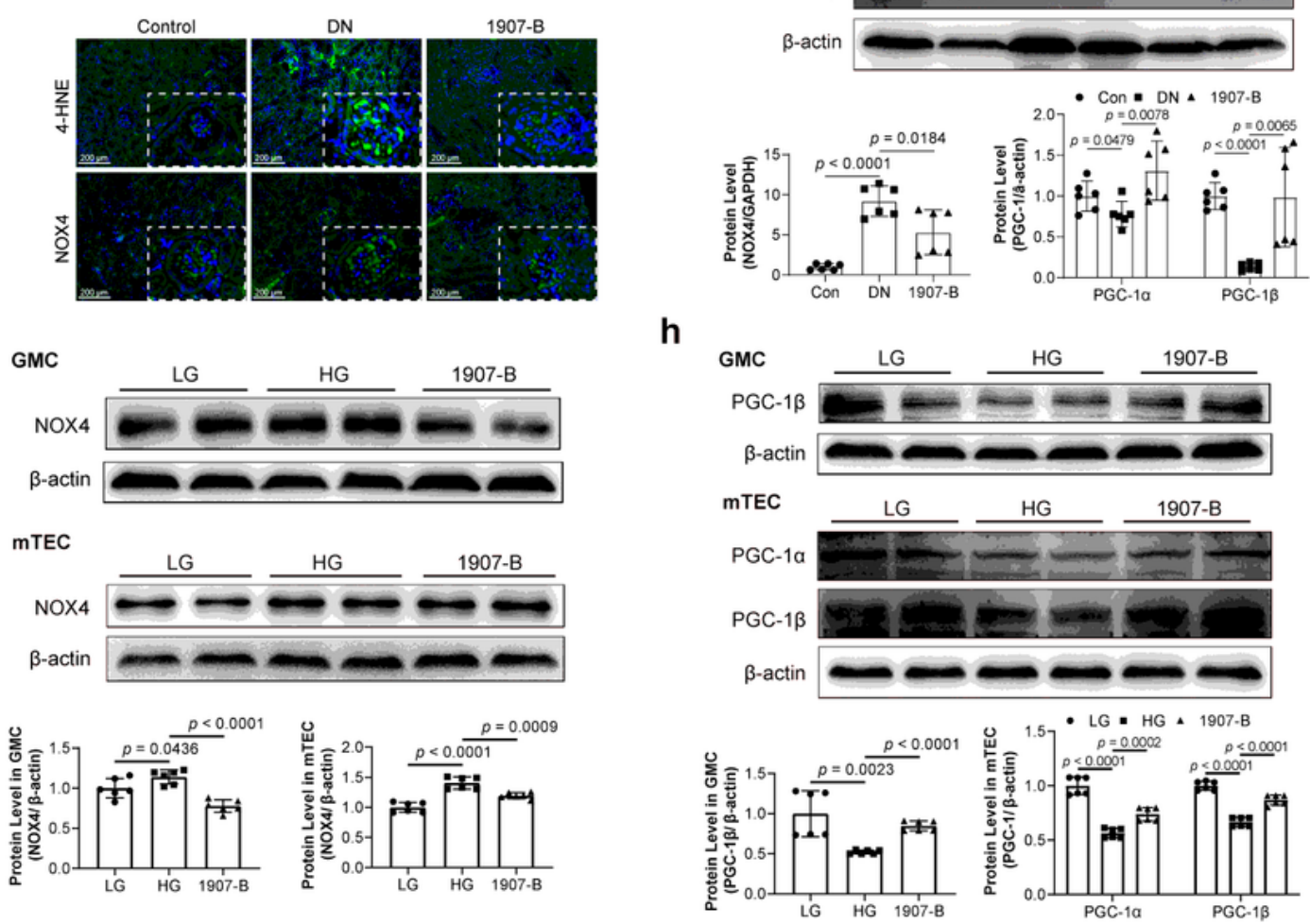

Figure 7

1907-B improves mitochondrial function and inhibits oxidative stress. a, GSEA pathway enrichment analysis of pathways related to mitochondrial function. b, the mRNA level of Ppargc1a, Ppargc1b, Sod1, Sod2, Cat, Hmox1, Uqcrc1 and Gpx1 were measured in the kidney samples of the indicated groups. c, The GSH content and activity of antioxidant stress related enzyme CAT and SOD in the kidney samples of the indicated groups. $\mathbf{d}$, Representative images of fluorescence staining of 4-HNE and NOX4 on kidneys from 
$\mathrm{db} / \mathrm{db}$ mice at indicated groups. ef, The expression level of key protein NOX4, PGC-1a and PGC-1 $\beta$ in the kidney of the indicated groups. $\mathbf{g}$-h, The protein expression level of NOX4, PGC-1a and PGC-1 $\beta$ in GMC and mTEC cells of the indicated groups.
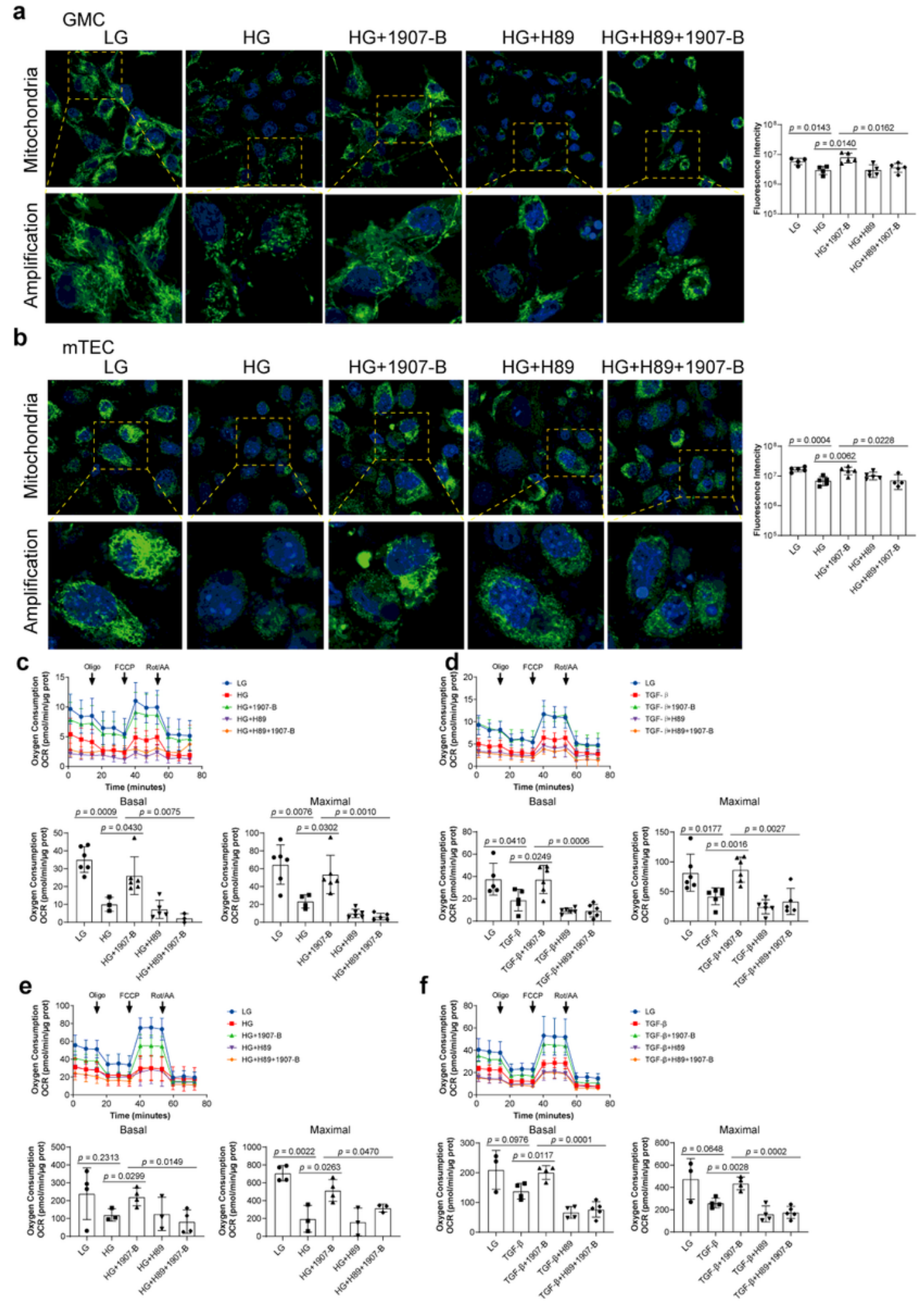

Figure 8 
1907-B enhances the mitochondrial function by targeting GLP-1R and GCGR and further activating downstream PKA pathway. a-b, Representative live cell confocal microscopy images showing GMC cells and $\mathrm{mTEC}$ cells of the indicated groups stained with Mito Tracker. $\mathbf{c}$ and $\mathbf{d}$, OCR during mitochondrial stress test of GMC cells under high glucose or TGF- $\beta$ treatment. $\mathbf{e}$ and $f, O C R$ during mitochondrial stress test of mTEC cells under high glucose or TGF- $\beta$ treatment.
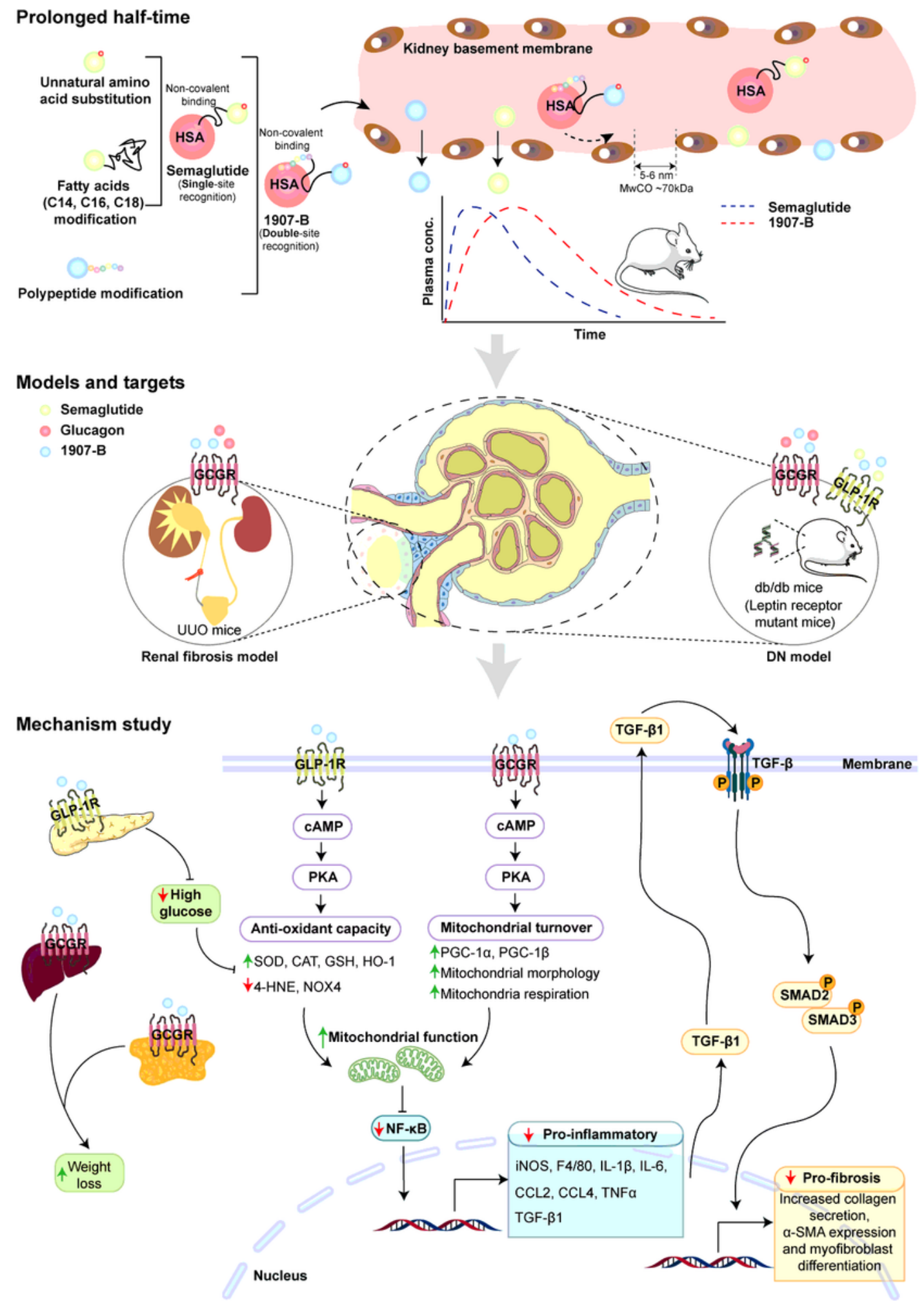

Figure 9 
Summary of 1907-B's ultralong-acting design and its action mechanism at targets organs. Compared with semaglutide, 1907-B exhibits more prolonged half-time due to introduction of C18 and "GGSGSG" in the side chain of Lys at position 10 of 1907. The dual-agonist activity of 1907-B acts at the GLP-1R and GCGR in the kidney to ameliorate the diabetic nephropathy in $\mathrm{db} / \mathrm{db}$ mice. Meanwhile, the GCGR agonist activity of 1907-B acts at renal tubules to attenuate renal fibrosis in UUO mice. Mechanistically, 1907-B could act at the GCGR in the liver and adipose tissue to induce increased body weight loss. Moreover, the GLP-1R agonist activity at pancreatic $\beta$-cells enhances insulin secretion to ameliorate whole-body glucose control. In the kidney, GLP-1R signaling could increase anti-oxidant capacity and lead to lower levels of oxidative stress, as well as GCGR signaling could improve mitochondrial maintenance. Altogether, these action in the kidney further reduces inflammation via NF-KB pathway and improves renal fibrosis via TGF$\beta$-SMAD pathway.

\section{Supplementary Files}

This is a list of supplementary files associated with this preprint. Click to download.

- 01132022supportinginformationfigureXtable.docx 